\title{
Host dietary specialization and neutral assembly shape gut bacterial communities of wild dragonflies
}

\author{
Rittik Deb $^{\text {Corresp., } 1}$, Ashwin Nair ${ }^{1,2}$, Deepa Agashe ${ }^{\text {Corresp. } 1}$ \\ 1 National Centre for Biological Sciences, TIFR, Bangalore, Karnataka, India \\ 2 Shanmugha Arts, Science, Technology \& Research Academy (SASTRA University), Thanjavur, Tamil Nadu, India \\ Corresponding Authors: Rittik Deb, Deepa Agashe \\ Email address: rittikd@ncbs.res.in, dagashe@ncbs.res.in
}

Host-associated gut microbiota can have significant impacts on host ecology and evolution and are often host-specific. Multiple factors can contribute to such host-specificity: (1) host dietary specialization passively determining microbial colonization, (2) hosts selecting for specific diet-acquired microbiota, or (3) a combination of both. The latter possibilities indicate a functional association and should produce stable microbiota. We tested these alternatives by analyzing the gut bacterial communities of 6 species of wild adult dragonfly populations collected across several geographic locations. The bacterial community composition was predominantly explained by sampling location, and only secondarily by host identity. To distinguish the role of host dietary specialization and host-imposed selection, we identified prey in the guts of three dragonfly species. Surprisingly, the dragonflies - considered to be generalist predators - consumed distinct prey; and the prey diversity was strongly correlated with the gut bacterial profile. Such host dietary specialization and spatial variation in bacterial communities suggested passive rather than selective underlying processes. Indeed, the abundance and distribution of $72 \%$ of bacterial taxa were consistent with neutral community assembly; and fluorescent-in-situhybridization revealed that bacteria only rarely colonized the gut lining. Our results contradict the expectation that host-imposed selection shapes the gut microbiota of most insects, and highlight the importance of joint analyses of diet and gut microbiota of natural host populations. 
1 Host dietary specialization and neutral assembly shape gut bacterial communities of wild

2 dragonflies

3

4 Authors: Rittik Deb ${ }^{1 *}$, Ashwin Nair ${ }^{1,2}$, Deepa Agashe ${ }^{1 *}$

5

6 Affiliation:

7 1. National Centre for Biological Sciences, TIFR, Bangalore, Karnataka, India

8 2. Shanmugha Arts, Science, Technology \& Research Academy (SASTRA University),

$9 \quad$ Thanjavur, Tamil Nadu, India

10

*Corresponding authors:

12 Rittik Deb

13 National Centre for Biological Sciences, TIFR, GKVK Campus, Bellary Road, Bangalore

14 560065, India. Email: debrittik@gmail.com, rittikd@ncbs.res.in

15 Deepa Agashe

16 Address: National Centre for Biological Sciences, TIFR, GKVK Campus, Bellary Road,

17 Bangalore 560065, Email: dagashe@,ncbs.res.in 


\section{ABSTRACT}

Host-associated gut microbiota can have significant impacts on host ecology and evolution and are often host-specific. Multiple factors can contribute to such host-specificity: (1) host dietary specialization passively determining microbial colonization, (2) hosts selecting for specific diet-acquired microbiota, or (3) a combination of both. The latter possibilities indicate a functional association and should produce stable microbiota. We tested these alternatives by analyzing the gut bacterial communities of 6 species of wild adult dragonfly populations collected across several geographic locations. The bacterial community composition was predominantly explained by sampling location, and only secondarily by host identity. To distinguish the role of host dietary specialization and host-imposed selection, we identified prey in the guts of three dragonfly species. Surprisingly, the dragonflies - considered to be generalist predators - consumed distinct prey; and the prey diversity was strongly correlated with the gut bacterial profile. Such host dietary specialization and spatial variation in bacterial communities suggested passive rather than selective underlying processes. Indeed, the abundance and distribution of $72 \%$ of bacterial taxa were consistent with neutral community assembly; and fluorescent-in-situ-hybridization revealed that bacteria only rarely colonized the gut lining. Our results contradict the expectation that host-imposed selection shapes the gut microbiota of most insects, and highlight the importance of joint analyses of diet and gut microbiota of natural host populations. interactions, Predator 
42 Host-associated gut microbial communities can have large impacts on host evolution. In turn, the 43 gut microbiome is affected by many factors including host genotype, environmental variation,

44

and host diet (Broderick \& Lemaitre, 2012; Colman, Toolson \& Takacs-Vesbach, 2012; McFallNgai et al., 2013; Kostic, Howitt \& Garrett, 2013; Engel \& Moran, 2013). However, it is not always clear whether these effects of host genotype, diet and environment reflect variation in the acquisition or the establishment step of microbial community assembly. Gut microbes are typically acquired from the mother, through social contact with conspecifics, or the diet; and they may either colonize the gut or fail to establish. At each step, various stochastic vs. deterministic, and neutral vs. selective processes determine community composition. For instance, a host may consistently acquire a specific set of microbes if they are maternally transmitted, or if the host is a dietary specialist. Within the host gut, microbial survival and growth dynamics may be primarily determined by stochastic neutral processes (e.g., based on initial abundance); or by deterministic and selective processes such as interactions with the host or with other microbes.

Gut-associated microbiomes can thus be considered as meta-communities (Sloan et al., 2007; Woodcock et al., 2007), where hosts represent local habitats that are colonized by microbes. Gut microbial community composition should thus depend on dispersal, subsequent filtering by the local environment (host), and successful establishment. Prior studies show that host diet can act as a major source of microbial colonizers, and thus can alter microbial dispersion into the host gut (Engel \& Moran, 2013), impact nutrient availability (for the microbes) in the gut (Laparra \& Sanz, 2010), and introduce new parasites thus inducing immune responses (Walk et al., 2010). In general, 
63 it is expected that generalist hosts are more likely to stochastically sample a wider range of

64 environmental microbes associated with a variable diet (in comparison with dietary specialists), as

65 observed for scavengers and omnivores (Yun et al., 2014; Yadav et al., 2015; Shukla et al., 2016).

66 This disruptive effect of dietary variation can be opposed by the strong host-imposed selection,

67 stabilizing gut bacterial community composition. Many studies have implicated such host-imposed

68 selection as a driver of gut bacterial community composition (Spor, Koren \& Ley, 2011; Engel \&

69 Moran, 2013; Antwis et al., 2017). For instance, host immune responses (Ley, Peterson \& Gordon,

70 2006; Charroux \& Royet, 2012; Broderick \& Lemaitre, 2012; Quigley, 2013) or a host-derived

71 protected niche inside gut crypts (Dillon \& Dillon, 2004; Kikuchi, Hosokawa \& Fukatsu, 2007;

72 Engel \& Moran, 2013) may allow only specific microbes to colonize the gut. In contrast, under

73 weak host selection, neutral processes such as ecological drift and microbial dispersal may strongly

74 drive community assembly (Hubbell, 2001; Rosindell, Hubbell \& Etienne, 2011), with each host's

75 microbiota functioning as a local community interacting with the larger meta-community outside

76 the host (Costello et al., 2009, 2012).

77

78 Although several studies have found substantial individual and population level variation in the microbiomes of wild-collected insects (Osei-Poku et al., 2012; Martinson, Douglas \& Jaenike,

80 2017; Sanders et al., 2017; Adair et al., 2018), the relative importance of various environmental

81 and host-specific factors in determining the composition and stability of gut bacterial

82 communities of natural animal populations remains unclear. We analyzed the gut bacterial and

83 prey community composition in natural populations of six dragonfly species (adults), sampled

84 from five locations in India (Fig 1A, and Table S1) without significant temporal variation (each

85 location sampled within 3-4 days). Dragonflies are generalist predators (Corbet, 2004) (i.e. 
86 predating on a diversity of prey items across its distribution); thus, we expected that they would

87 consume diverse insect prey across locations and host species. In turn, this dietary diversity

88 should be associated with diverse gut microbial communities. Previously, we found that the

89 culturable fraction of gut bacterial communities of adult dragonflies varied significantly as a

90 function of host species, location, and sampling time (Nair \& Agashe, 2016). Here, we built upon

91 this work by sampling more dragonflies, identifying most gut-associated bacteria using $16 \mathrm{~S}$

92 rRNA amplicon sequencing, and analyzing host diet by sequencing the cytochrome c oxidase 1

93 gene (COX1) from gut contents. We then quantified the spatial stability of host-associated gut

94 microbiomes; tested whether bacterial diversity correlates with host diet diversity; and quantified

95 the relative importance of neutral processes driving bacterial community assembly.

2. MATERIALS AND METHODS

98

Sample collection and storage

We collected adult dragonflies from five different sampling sites across India (Fig 1A and Table

S1A), focusing on the species Orthetrum pruinosum, Orthetrum sabina, Pantala flavescens, all

from the family Libellulidae, order Odonata (http://indiabiodiversity.org/ for species

identification). We also sampled 3 other dragonfly species Trithemis aurora, Urothemis signata, and Zygonyx iris (family: Libellulidae), from one geographic location - Shendurney (Fig 1A,

Table S1B) (for shendurney samples only permit obtained from Kerala Forest Department, permit no. WL 10-3781/2012 dated 18/12/2012, and GO (RT) No. 376/2012/F and WLD dated 26/07/2012). We mainly sampled during the monsoon and post-monsoon season (October - early

January) when dragonfly populations are at their peak (except for 3 individuals of species $O$. 
109

110

111

112

113

114

115

116

117

118

119

120

121

122

123

124

125

126

127

128

129

130

131

sabina that were collected during summer from Agumbe region). We sampled each location once within a span of 3-4 days during the monsoon and post monsoon (Table S1). We caught individuals using butterfly nets in open grounds, near natural water bodies, or waterlogged paddy fields. We collected three sets of samples to determine (a) the composition of gut bacterial communities (using amplicon sequencing of 16S rRNA), (b) the absolute abundance of focal bacteria (using qPCR) and to localise them in the gut (using fluorescent in-situ hybridization, FISH), and (c) dragonfly diet (using amplicon sequencing of cytochrome c oxidase 1 gene $(\mathrm{COX} 1))$.

(a) To determine the composition of gut bacterial communities, we surface sterilized each dragonfly (see Table S1 for sampling details) using 70\% ethanol and stored it in a $1 \mathrm{x} 1 \mathrm{ft}$. mesh cage. Within 4-6 hours of collection, we paralyzed dragonflies using a $4^{\circ} \mathrm{C}$ cold shock and dissected them in phosphate-buffered saline (PBS) using sterilized dissection tools. We stored dissected guts in $1.5 \mathrm{ml}$ centrifuge tubes containing $100 \%$ molecular grade ethanol. We stored the remaining dragonfly bodies separately in $100 \%$ ethanol for subsequent identification based on a morphological key using an online resource (http://indiabiodiversity.org/). After bringing samples to the laboratory, we stored samples at $-20^{\circ} \mathrm{C}$ until further processing. For collections in Bordubi and Nagpur, we could not dissect dragonflies in the field, so we stored them in 100\% ethanol immediately after capture. We dissected these samples also within 4-6 hours of capture.

(b) To estimate absolute gut bacterial abundance (using qPCR) and to localize bacteria in dragonfly guts (using FISH), we collected and isolated dragonflies in $50 \mathrm{ml}$ Falcon tubes for 4-6 hours (see Table S1 for sampling details). Such isolation allowed the dragonflies to defecate and remove large and probably indigestible food particles from the gut, which can cause difficulties during microtome sectioning of gut tissues. For dragonflies collected for qPCR, we dissected the 
132 entire gut in PBS and stored the guts in $100 \%$ ethanol. For FISH, we dissected the gut in PBS,

133 divided each gut into three sections (foregut, midgut, and hindgut), and stored each section

134 separately in $100 \%$ ethanol. We brought the dragonfly bodies and the guts back to the laboratory

135 and stored them at $-20^{\circ} \mathrm{C}$.

136 (c) To analyze the dragonfly diet, we again collected individuals of three of the well-sampled

137 dragonfly species used for gut bacterial community analysis (O. pruinosum, $O$. sabina, and $P$.

138 flavescens) (Fig 1A, see Table S1 for sampling details). We dissected out the entire guts in PBS.

139 We made an incision in the gut wall and centrifuged the gut for 30 seconds at $1000 \mathrm{rpm}$ to

140 separate gut contents from the gut tissue. We only stored the gut contents in Eppendorf tubes in

$141100 \%$ ethanol. We brought the dragonfly bodies and the guts back to the laboratory and stored

142 them at $-20^{\circ} \mathrm{C}$ until further processing.

Amplicon sequencing to determine gut bacterial and diet composition

145 We determined the gut bacterial community for a total of 47 dragonflies from different species 146 and geographical locations (Fig 1A, Table S1). We washed each stored gut sample (intact gut 147 along with its contents) thrice in fresh $100 \%$ molecular grade ethanol followed by three washes

148 in PBS. We homogenized the tissue in liquid nitrogen using single-use sterile pestles and extracted DNA using the Wizard ${ }^{\circledR}$ Genomic DNA Purification Kit (Promega Corporations,

150 Wisconsin, Madison, USA). We modified the manufacturer's protocol as follows: we added $600 \mu \mathrm{l}$ of nuclei lysis solution (10mM EDTA) per $100 \mathrm{mg}$ tissue and incubated first at $80^{\circ} \mathrm{C}$ for 20

$152 \mathrm{~min}$, and then at $65^{\circ} \mathrm{C}$ for $30 \mathrm{~min}$. We cooled the samples to $55^{\circ} \mathrm{C}$, added $20 \mathrm{mg} / \mathrm{ml}$ proteinase,

153 and again incubated at $55^{\circ} \mathrm{C}$ for 3 hours. To precipitate degraded protein, we added protein

154 precipitation solution and left the sample on ice for $30 \mathrm{~min}$. We centrifuged the lysate at $14000 \mathrm{~g}$ 
155 for $10 \mathrm{~min}$ and precipitated the supernatant with isopropanol. We washed the resulting pellet

156 with $80 \%$ ethanol twice, then dried and suspended it in $40 \mu 1$ ultrapure nuclease-free water. We

157 quantified DNA in a Nano-Drop (Nano-drop 2000, Thermo Fisher Scientific Inc., Wilmington,

158 USA). We also generated negative controls, where we prepared 16S rRNA libraries without

159 adding sample DNA, and quantified DNA after each PCR step using a highly sensitive Qubit 3

160 fluorometer (Invitrogen, ThermoFisher Scientific Inc.) (as described in Phalnikar, Kunte \&

161 Agashe, 2018). However, we did not detect any DNA in any of the blank samples; in contrast,

162 we detected high concentrations of DNA $(85-300 \mathrm{ng} / \mu \mathrm{l})$ for all gut microbe samples. We also

163 checked the integrity of the DNA (for gut samples) by running $1 \mu \mathrm{g}$ on a $0.8 \%$ agarose gel. For

164 each sample, we used 50 ng DNA to PCR-amplify the V3-V4 hypervariable region of the

165 bacterial 16S rRNA gene, using ExTaq (TaKaRa). The PCR primers contained tag sequences

166 complementary to the Illumina sequencing adapter and index primers from the Nextera XT Index

167 kit V2. We tested amplicons for quality and sequenced them (250 bp paired-end) on the Illumina

168 MiSeq platform (Illumina, San Diego, CA, USA) using standard Illumina forward and reverse

169 primers. Sequencing was performed by Genotypic Technology Pvt. Ltd., Bangalore, India.

170

171 For host diet analysis, we implemented (with few modifications) a previously described method

172 that was used to estimate diet diversity in insectivorous bats (Zeale et al., 2011), and recently in

173 odonates (Kaunisto et al., 2017). A recent study by Kamenova and colleagues (2017) showed

174 that prey DNA remains relatively intact inside the gut of a predatory carabid beetle (Pterostichus

175 melanarius) for at least 3-5 days. Assuming a similar prey retention time in dragonfly guts, we

176 thus expected that our analysis would reflect a 3-5 day snapshot of dietary diversity in each

177 dragonfly. In brief, we targeted the variable region of the COX1 gene - found in all insects - to 
178 estimate insect prey diversity from the gut contents of captured dragonflies. Prior studies suggest 179 that COX1 primer sites (as used in Zeale et. al. (2011)) are often less conserved, leading to 180 unreliable amplification, especially in animal groups such as nematodes (Deagle et al., 2014).

181 However, currently COX1 has the best database of barcode sequences with taxonomically 182 verified organisms, which is critical for diet studies. We sampled a total of 45 dragonflies 183 representing three species; as well as a phytophagous butterfly larva (Hasora sp.) as a positive 184 control. The phytophagous butterfly provided an ideal control, since we expected amplification 185 of only the host DNA in this sample. Any non-host reads would indicate non-specific 186 amplification of contaminants during sequencing. We expected that if our classification were

187 188 189

correct, it would classify all the sequences coming from this sample as Hasora sp. We extracted dragonfly gut contents after removing the host tissue (as described earlier) and then extracted DNA from the gut contents. For our control sample, we homogenized the tissue in liquid nitrogen using single-use sterile pestles and used then further processed it for DNA extraction. We extracted DNA using the Wizard ${ }^{\circledR}$ Genomic DNA Purification Kit (Promega Corporations, Wisconsin, Madison, USA) with the following modifications. We lysed cells at $65^{\circ} \mathrm{C}$ in nuclei lysis solution with $10 \mathrm{mM}$ EDTA, followed by an overnight proteinase K treatment. We precipitated DNA overnight at $-20^{\circ} \mathrm{C}$, suspended the final pellet in $20 \mu 1$ nuclease-free water, and checked the concentration and integrity of the DNA. For further analysis, we chose samples showing intact bands on an agarose gel $(\mathrm{n}=28$ dragonflies, and 1 butterfly larva; Table S1). We designed custom primers - containing Illumina ITS barcodes for multiplexing - to target the COX1 variable region (using references from Zeale et al., 2011). The forward primer sequence was 5'-TCGTCGGCAGCGTCAGATGTGTATAAGAGACAG AGATATTGGAACWTTATATTT TATTTTTGG-3', and the reverse primer was 5'- 
201

202

203

204

205

206

207

208

209

210

211

212

213

214

215

GTCTCGTGGGCTCGGAGATGTGTA TAAGAGACAG-

WACTAATCAATTWCCAAATCCTCC-3'. We used 200 ng DNA from each sample to amplify the target COX1 region with High Fidelity Phusion polymerase (Thermo scientific). We purified the samples using the Qiagen PCR purification Kit (Qiagen) and checked the product for amplicon size and concentration (as mentioned above). We did not use blocking primers (Vestheim \& Jarman, 2008; De Barba et al., 2014) or PNA (thermally more stable) (Chow et al., 2011; Terahara et al., 2011) to amplify prey DNA because we did not know the prey diversity in dragonflies, and some dragonflies are known predators of other (closely related) dragonflies.

These two factors made it difficult to design blocking primers or PNA that would block only host DNA (Piñol et al., 2014). We also prepared blank samples (as negative controls) and tested for DNA concentration in these blank samples to check for possible contamination (as described above). Concordant to our previous extraction, we did not find any detectable DNA

concentration in our blank samples. Finally, for the dragonfly gut content and the positive control (Hasora sp. larva) sample, we prepared sequencing libraries using the Nextera XT v2 Index Kit (Illumina, U.S.A.) and sequenced them on the MiSeq platform (250 bp paired-end). Sequencing was performed by Genotypic Technology Pvt. Ltd., Bangalore, India.

We processed amplicon sequencing data using QIIME (Caporaso et al., 2010) using conservative protocols to reduce errors. After demultiplexing and removing barcodes and primer sequences, we filtered and trimmed reads for sequence length and quality score $(q>20)$ using default QIIME parameters. We used Fast-QC ("Babraham Bioinformatics - FastQC A Quality Control tool for High Throughput Sequence Data") to check read quality and presence of barcodes or primers in the processed data (see Fig S1 for workflow). Finally, we paired the forward and reverse reads to 
224 generate a total of 30 million high-quality paired-end reads for the $16 \mathrm{~S}$ rRNA gene, with an

225 average of 169,000 reads per sample (range: 8,000-900,000, post quality filtering 62000-599000,

226 Fig S2). We classified reads into Operational Taxonomic Units (OTUs) at the 97\% similarity

227 level using UCLUST (Edgar, 2010), using both open and closed reference OTU picking. For

228 OTU picking, we set both "maxaccepts" and "maxrejects" values to 0 , to ensure an exhaustive

229 search. We used the GreenGenes 16S rRNA ribosomal gene database version gg_13_8 (DeSantis

230 et al., 2006) to assign taxonomy to each representative OTU. We removed chimeric sequences

231 using Chimeraslayer (Haas et al., 2011) and also removed unassigned, chloroplast, and

232 mitochondrial sequences to generate the final ".biom" files for OTU picking. We normalized

233 closed referenced OTUs by bacterial 16S rRNA copy number using the software PICRUSt

234 (Phylogenetic Investigation of Communities by Reconstruction of Unobserved State, version

235 1.0.0) (Langille et al., 2013). For insect COX1 amplicons, we obtained a total of 2.1 million

236 reads (average 70,000 and range 29,000-115,000 reads per sample, Fig S2). We picked OTUs

237 using similarity cut off (97\%) described in Hebert et al. (2003) for COX-1 region and used the

238 Barcode of Life Database v4 (Ratnasingham \& Hebert, 2007) (“"bold: Interface to Bold Systems

239 API version 0.9.0 from CRAN") to assign taxonomy to each OTU. We removed chimeric

240 sequences and checked the precision of our sequencing and OTU assignment using our control

241 sample (Hasora sp. butterfly larva), where $97.4 \%$ of the reads were correctly classified to a

242 single OTU assigned to Hasora sp. We removed the most abundant Odonate OTU from each

243 sample since it likely represented host amplicons, although this could also remove potential cases

244 of conspecific predation (Corbet, 2004) (Fig S3). However, this step was essential because

245 several studies examining host diet using host gut or in faecal samples have reported high 
246 proportion of host DNA in their sequencing output (Piñol et al., 2014; De Barba et al., 2014;

247 Kaunisto et al., 2017).

248

249 We removed potentially erroneous OTUs (as described in Huse et al., 2010) from both amplicon 250 datasets by implementing three OTU filters. 1) Pruned community: retaining all OTUs with at 251 least $0.005 \%$ relative abundance across the entire dataset, to minimize impacts of sequencing 252 errors (Bokulich et al., 2013); 2) Dominant community: retaining all OTUs with at least 5\% 253 relative abundance in at least one sample; 3) Minimally pruned community: retaining all OTUs 254 with at least 20 reads per OTU per sample, to obtain a conservative estimate of OTUs with

255

256

257

258

259

260

261

262

263

264

265

266

267 sufficient read support. We separately applied each filter to the full dataset and then recalculated the relative abundance of OTUs for subsequent analysis.

\section{Statistical analysis}

We performed all statistical analysis in the R statistical software version 3.3.4 (R Core Team, 2013), considering each OTU as the basic unit of comparison regardless of taxonomic placement. To estimate the sampling depth at which the community richness saturated, we performed a rarefaction analysis with the Pruned Community in QIIME (Caporaso et al., 2010). We subsampled reads to simulate varying sampling depth (100-2500 reads per sample) and calculated Faith's phylogenetic diversity (Faith's PD) at each depth. We then estimated the sampling depth at which PD saturated, as an indicator of sufficient sampling. We also rarefied the community to its lowest sampling depth and subsampled the community 100 times. We calculated the mean rarefied community and its standard deviation, and also used these data for 
268 analysis. However, in the results section, unless specifically mentioned all analysed communities

269 are unrarefied communities.

270

271 We analyzed community structure (relative abundance of OTUs) across samples using Ward's

272 hierarchical agglomerative clustering (Murtagh \& Legendre, 2014). We tested the impact of host

273 species and geographical location on the gut bacterial community composition using

274 permutational ANOVA (PERMANOVA, in the R package vegan (Dixon, 2003; Oksanen et al.,

275 2017) using function adonis) with 10,000 permutations. We also performed PERMANOVA

276 using library size as an additional factor, to understand whether library size influenced the

277 outcome (Weiss et al., 2017). We used the R package "Caret” (Kuhn, 2008) to remove near-zero

278 variance in the data (i.e. OTU vectors with little or no variance). We performed this step because

279 these vectors provide no information, but did not allow our downstream analysis (CAPdiscrim

280 function) to proceed. To visualize sample clustering based on bacterial composition across

281 treatments, we calculated Bray-Curtis distances between samples and performed Canonical

282 Analysis of Principal Coordinates based on Discriminant Analysis (CAPdiscrim (Anderson \&

283 Willis, 2003)) using the R package "Biodiversity R" (Kindt \& Kindt, 2017). We tested the

284 significance of clustering and estimated classification success by permuting the distance matrix

2851000 times and calculated P value. We plotted the two dominant linear discriminants (LD) to

286 visualize data classification. For each cluster, we drew ellipses reflecting $95 \%$ confidence

287 intervals using the function "Ordiellipse" in the R package "Vegan" (Dixon, 2003; Oksanen et

288 al., 2017). This analysis was also perform on the mean rarefied community for comparison. 
290 To estimate bacterial or prey OTU richness for each dragonfly sample, we generated a presence-

291 absence matrix from the relative abundance of each OTU. We used the final ".biom" table to

292 identify shared OTUs across samples, and to calculate OTU richness per sample, and $\alpha$ diversity

293 (Shannon's diversity index, a measure of OTU richness and evenness per sample), $\beta_{\mathrm{w}}(\mathrm{a}$

294 comprehensive measure of the number of unique OTUs per sample (Koleff, Gaston \& Lennon,

295 2003)) using the R package Vegan (function "diversity" for Shannon's index, and "betadiver"

296 for $\beta_{\mathrm{w}}$ diversity) (Dixon, 2003; Oksanen et al., 2017) and $\beta_{\text {Aitchison }}$ diversity (measure of $\beta$ -

297 diversity for compositional data using R-package robCompositions (Martino et al., 2019)). We

298 also measured the $\alpha$ diversity and $\beta_{\mathrm{w}}$ for mean, $+1 \mathrm{SD}$ and $-1 \mathrm{SD}$ rarefied communities for

299 comparison. We tested the effect of host species identity, and sampling location on bacterial

300 OTU richness using a generalized linear model (GLM) with Poisson errors (using the R package

301 Stats (R Core Team, 2013)). We also performed Kruskal Wallis' test followed by post-hoc

302 Conover test (using the R package PMCMR (“CRAN - Package PMCMR”) to compare between

303 bacterial richness and diversity with the prey richness and diversity of the three main dragonflies.

Testing models of bacterial community assembly in dragonfly guts

306 If the gut community of a host is under weak selection, we expected it to be predominantly

307 neutrally assembled. To test whether a neutral model of community assembly could explain the

308 observed distribution of bacterial communities across hosts, we fitted a neutral distribution

309 model (Sloan et al., 2006; Woodcock et al., 2007) to the bacterial communities observed in

310 dragonfly hosts from a specific location. The model is based on Hubbell's model of the neutral

311 theory of biodiversity (Hubbell, 2001), but applies to large communities, such as a complex

312 microbial community. For model fitting (using the open reference pruned community), we 
313 followed the approach used by Burn et al. (2016). We assumed that each individual dragonfly

314 gut houses a local community with numerous bacterial species (OTUs) whose members are

315 drawn from a larger metacommunity, comprised of bacteria present across all dragonfly

316 individuals collected from a specific geographic location. The model uses the following

317 parameters: (a) population size of each OTU in the local and metacommunity (estimated using

318 the number of reads) (b) the relative abundance of each OTU. Using these, the model estimates

319 the migration rate or dispersion probability $(\mathrm{m})$ for each OTU. In the event of an individual

320 bacterium's death, $\mathrm{m}$ is the probability that it will be replaced via dispersal from the

321 metacommunity, rather than reproduction within the local community. The relationship between

322 the abundance of each OTU in the metacommunity and its occurrence across local communities

323 is informative for understanding the processes driving community assembly (Sloan et al., 2006).

324 For each metacommunity, we fit a $\beta$-distribution to the relationship between OTU occurrence

325 and abundance (using the script published by Burns et al. (2016); please see Supplementary

326 Code 1 published in Burns et al. 2016 (https://media.nature.com/original/nature-

327 assets/ismej/journal/v10/n3/extref/ismej2015142x4.txt) ; R-packages used: vegan (Dixon, 2003;

328 Oksanen et al., 2017), minpack.lm (Elzhov et al., 2010), Hmisc (Harrell \& Dupont, 2008)). We

329 checked the fit using non-linear least squares in R and estimated 99\% (and 95\%) confidence

330 intervals (CI) around the fit using binomial proportions. We then compared the proportion of

331 OTUs that were neutrally distributed across sites and hosts. Under neutral assembly, a highly

332 abundant OTU should occur in many hosts. If an OTU occurs at a higher frequency in a host

333 than expected from its abundance in the metacommunity (comprised of OTUs from all hosts),

334 this indicates positive selection for those bacteria (presumably by the host). Similarly, if an OTU

335 is very abundant in the metacommunity but occurs in only a few host individuals, this indicates 
336 negative selection against the OTU. Finally, we compared taxonomic diversity (Clarke \&

337 Warwick, 1998; Fierer, Bradford \& Jackson, 2007; Morrow et al., 2015) between the groups of

338 bacteria that were inferred to be neutrally distributed or positively or negatively selected by

339 hosts. If hosts selected for a specific functional association (and this functionality is

340 phylogenetically conserved in bacteria), we expected that bacterial OTUs experiencing positive

341 host selection should have lower taxonomic diversity compared to neutrally assembled bacteria.

342 To estimate the fit of each model, we calculated generalized R square $\left(R^{2}\right)$ (as suggested by

343 Burns et al. (2016)). We also fitted a binomial distribution model that assumed that the local

344 communities were only a subset of the meta-communities without any dispersion and drift (as

345 suggested by Burns et al. (2016) and Sloan et al. (2007)). We compared the fit of both binomial

346 as well as Sloan's neutral models using $\mathrm{R}^{2}$ and Akaike information criterion (AIC) (as

347 suggested by Burns et al. (2016)).

349 We generated two sets of models; (1) for each dragonfly species sampled at a specific location, 350 and (2) pooling all dragonfly species sampled in each location. The first set allowed us to infer

351 patterns of gut bacterial community assembly for each dragonfly species, but with low sample

352 sizes (Table S1). The second set allowed us to infer general patterns of gut bacterial assembly 353 across dragonflies, with a larger sample size.

354

Localizing bacteria in dragonfly guts

356 We used fluorescent in-situ hybridization (FISH) to determine the location of bacteria inside

357 dragonfly guts. We hypothesized that if there is a functional association between host and

358 bacteria, bacterial cells should be housed in specific crypts or inside columnar cellular folds in 
359 the host gut (as reported in previous studies by Barrow et al., 1980; Fuller \& Turvey, 1971). We

360 performed FISH for three dragonfly species (O. sabina, O. pruinosum, and $P$. flavescens; $\mathrm{n}=5$

361 individuals per species). We used a universal eubacterial probe ([Alexa-488] 5'-

362 GCTGCCTCCCGTAGGAGT-3' (Da Silva et al., 2015)) and a Wolbachia-specific probe

363 ([ALEXA-647] 5'-CTTCTGTGAGTACCGTCATTATC-3' (Le Clec'h et al., 2013)) (Sigma-

364 Aldrich-Merck, Missouri, USA) to stain the specific gut bacteria. We used DAPI (4', 6-

365 diamidino-2-phenylindole) staining to visualize host cell nuclei. We followed the COLOSS

366 protocol (Kwong et al., 2013) with a few modifications. Before the assay, we rehydrated guts

367 and fixed them in Carnoy's fixative for 96 hours. To reduce autofluorescence, we used peroxide

368 treatment for 72 hours and replaced the water inside host tissues using absolute ethanol and

369 xylene washes as per the COLOSS protocol. Finally, we embedded samples in liquid paraffin

370 using plastic moulds to create paraffin blocks. We sliced the blocks into $10 \mu \mathrm{m}$ transverse

371 sections using a Leica manual microtome (Leica Microtome 2125 RTS, Wetzlar, Germany)

372 using disposable blades (Low profile blade 819, Leica). For each species and each part of the gut

373 (foregut, midgut, and hindgut), we obtained 5 sections per probe for each of 5 individuals. We

374 mounted sections on Fisherbrand Superfrost Plus microscope slides (Thermo Fisher Scientific,

375 Wilmington, USA), and heated off the paraffin in an oven at $65^{\circ} \mathrm{C}$. We washed with Xylene

376 (three-minute wash, thrice), absolute ethanol (three-minute wash, thrice), and double-distilled

377 water (once) before hybridization. We dissolved $0.5 \mu \mathrm{L}$ of fluorescent probes in $500 \mu \mathrm{L}$

378 hybridization buffer and stained gut tissue sections in a dark chamber for 8-10 hours at room

379 temperature with the respective bacteria-specific probe. We then stained sections with DAPI for

38020 min to visualize host cell nuclei. We applied DABCO-glycerol (antifade agent), sealed the

381 sections with coverslips, and stored them at $4^{\circ} \mathrm{C}$ in the dark. For each species and each part of the 
382 gut (foregut, midgut, and hindgut), we obtained 5 sections per probe for each of 5 individuals.

383 Though we lost many sections during the multiple washes, we retained at least 2

384 sections/dragonfly/species/probe for final analysis. We imaged sections using a Zeiss 510 Meta

385 confocal microscope (Oberkochen, Germany) and analyzed images using Image-J software

386 (Version 1.6.0-24, 64-bit version). As a positive control, we probed heat-fixed bacterial smears

387 (5 slides of Bacillus thuringiensis from laboratory culture) with the eubacterial probe (following

388 the above protocol). As a negative control, we probed dragonfly tissue sections (3 midgut tissue

389 sections of each host species) using fluorophore-free eubacterial and Wolbachia probes, before

390 hybridizing with the probes containing active fluorophores. We expected that fluorophore-free

391 probes would get attached to the bacterial cells and prevent the fluorophore-attached probes from

392 binding with bacterial DNA.

393

394

Quantitative PCR to validate the abundance of specific gut bacteria

395

396

397

398

399

400

401

402

403

404

To estimate the abundance of eubacteria and Wolbachia (a common insect-associated bacterial genus), we performed quantitative PCR (qPCR) on bacterial DNA extracted from 9 dragonfly guts (three individuals each of $O$. sabina, O. pruinosum and P. flavescens). We used previously reported primers (Heddi et al., 1999): universal Eubacterial primers, forward 5'-

AGAGTTTGATCATGGCTCAG-3' and reverse 5'-TACCTTGTTACGACTTCACC-3'; and Wolbachia specific primers, forward 5'-CGGGGGAAAAATTTATTGCT-3', reverse 5'-

AGCTGTAATACAGAAAGTAAA-3'. To normalize bacterial abundance to host tissue, we used previously described Odonate-specific primers for the 28S gene (forward: 5'ACCATGAAAGGTGTTGGTTG-3' and reverse: 5'-ATCTCCCTGCGAGAGGATTC-3') (Dijkstra et al., 2014). All primer pairs had amplification efficiencies greater than $90 \%$. We ran 
405 three sets of PCR for each sample (total $10 \mu \mathrm{L}$ reaction volume), using $10 \mathrm{ng}$ of host gut DNA, 8

$406 \mu \mathrm{L}$ SYBR green PCR master mix (Thermo Fisher Scientific, Wilmington USA), and the

407 appropriate primers (200 $\mathrm{nM}$ each). We added reaction mixes in 384 well microplates (Corning,

408 New York, USA) and monitored amplification in a ViiA ${ }^{\text {TM }} 7$ Real-Time PCR System (Thermo

409 Fisher Scientific, Wilmington USA). We used the following cycle conditions: $95^{\circ} \mathrm{C}$ for $30 \mathrm{~s}, 40$

410 cycles of $95^{\circ} \mathrm{C}$ for $60 \mathrm{~s}, 56^{\circ} \mathrm{C}$ for $60 \mathrm{~s}, 72^{\circ} \mathrm{C}$ for $60 \mathrm{~s}$, and extension at $72^{\circ} \mathrm{C}$ for $5 \mathrm{~min}$. We

411 calculated threshold cycle values $\left(C_{T}\right)$ for each sample. We used the $C_{T}$ value of each host-

412 specific gene to estimate the $\Delta \mathrm{C}_{\mathrm{T}}$ values. Finally, we plotted these values for all the three host

413 dragonflies for comparison.

\section{RESULTS}

The gut bacterial community of dragonflies

Initial rarefaction analysis (collector's curve) revealed that our sampling depth was sufficient to

419 determine the bacterial community composition in all but one sample (one Orthetrum sabina sample), which we excluded from further analysis (Fig S4). Our PERMANOVA analysis revealed that library size did not influence the gut bacterial community composition (Table S2 A and B). As all samples showed sufficient sampling depth, we used the original dataset (nonrarefied) for our entire analysis. Moreover, McMurdie et al. (2014) have shown that rarefied

424 datasets can lead to an erroneous measurement of species abundance, especially if a species is differentially abundant across sample communities. However, in relevant sections, we have also

426 highlighted the results of our analysis of the mean rarefied (mean of 100 iterations) community 427 for comparison. We separately analyzed a total of six sets of non-rarefied bacterial communities 
428 across all dragonfly species (6) and locations (5), generated using either closed or open-reference

429 OTU picking and implementing three OTU filtering thresholds: 1) pruned community (576

430 OTUs open reference), 2) dominant community (59 OTUs open reference), and 3) minimally

431 pruned community (2599 OTUs open reference). All sets showed comparable results, but here

432 we focus on the pruned and dominant open referenced sets unless mentioned otherwise.

433 Corresponding results for other sets as well as rarefied communities are given in the

434 supplementary material.

435

436 We found an average of 188 OTUs per sample, most belonging to Proteobacteria (88\%),

437 Firmicutes (9.8\%), Actinobacteria (1.8\%) and Bacteroidetes (0.4\%) (Figs 1B \& 1C). At the

438 family level, Rickettsiaceae - comprising of three Wolbachia OTUs - were most abundant,

439 although this high abundance was limited to dragonflies from the genus Orthetrum (Fig 1C). In

440 other host genera, especially P. flavescens, OTUs from the family Enterobacteriaceae were more

441 abundant. Overall, we observed substantial variation in the relative abundance of OTUs across

442 host species, as well as across host individuals (Fig 1C, Fig S5: all species, open referenced,

443 dominant community).

444

445 Host species and sampling location shape gut bacterial community composition

446 We found that both host species and sampling location significantly affected the composition of

447 the dominant gut bacterial community (PERMANOVA; Table 1; see Table S3 for other

448 community sets) across the three best-sampled dragonflies (Table S1A). Linear discriminant

449 analysis (constrained by the group, CAPdiscrim) to visualize clustering supported these results,

450 showing strong separation in bacterial communities across host species and sampling location 
451 (Fig 2A-B, S5, and S6; see Fig S7A-B for mean rarefied community). Interestingly, location

452 explained a considerably larger proportion of variation in gut bacterial communities (26\%; Table

453 1, Table S3) compared to host species alone (9\%), suggesting that environmental factors (such as

454 local microbial community and prey community, soil $\mathrm{pH}$, or rainfall) have a stronger impact on

455 community composition (Fig 2A-B, S6A-B, S7A-B). This pattern was consistent irrespective of

456 the OTU filtering or referencing methodology used (open or closed; Table S3). When we

457 restricted our analysis to the geographic location Shendurney, where we had sampled a total of 5

458 different dragonfly host species (Table S1B), we found a similarly weak yet significant impact of

459 host species on dragonfly gut bacterial composition (Table 1B and Fig. 2C; Fig S7C for rarefied

460 mean community).

461

462 These patterns were also mirrored in the number of shared bacterial taxa across dragonflies. Of

463 the 576 OTUs detected in total across all dragonflies, 206 OTUs $(\sim 36 \%)$ were found in all host

464 species (Fig S8E). The percentage of shared OTUs increased to 64\% (366 OTUs out of 571

465 OTUs) when we considered only the three well-sampled dragonflies (Table S1A). The

466 congeneric dragonflies $O$. pruinosum and $O$. sabina, which harbored similar bacterial

467 communities (Fig 2A), also shared the maximum number of bacterial OTUs (407 shared OTUs,

$468 \sim 71 \%$, of which 34 OTUs were unique to the genus Orthetrum; Fig S8E). Only $25 \%$ of the

469 OTUs (145 out of 576 OTUs) were shared across locations (Fig S8F) when we considered all

470 dragonfly samples. This proportion increased marginally $(30 \%, 175$ out of 575 OTUs) for the

471 subset of well-sampled dragonflies (Table S1A). Finally, for each explanatory factor,

472 classification analysis based on gut bacterial composition categorised significant proportions of

473 samples correctly into the respective groups (for well-sampled dragonflies: Fig 2A-B, Tables 
474 S4A-B (open reference dominant community) and S5A-B (closed reference dominant

475 community); for 5 dragonfly species at Shendurney: Fig 2C, Tables S4C (open reference

476 dominant community) and S5C (closed reference dominant community)).

477

478 Our amplicon sequencing data had revealed that families Rickettsiaceae and Enterobacteriaceae

479 were most abundant in the gut of dragonflies (Fig 1C). We tested whether removing these two

480 dominant families altered the impact of host species and sampling location on the observed

481 community composition. We found that the results were robust to their removal (Fig S12).

482 Conversely, the abundance of OTUs from these families was influenced both by host species and

483

484

485

486

487

488

489

490

491

492

493

494

495

496

sampling location (Figs 1C and S13; Tables S6 and S7), mirroring our results for the full dataset.

Interestingly, we found an extremely high abundance of bacteria from the family Rickettsiaceae

in the gut tissues of samples from the genus Orthetrum (Fig 1C, Fig S13). We validated this

observation using qPCR, which revealed that Wolbachia (which belongs to this family) was

much more abundant in the genus Orthetrum compared to P. flavescens (Fig S14).

Despite the significant effects of host species and location on community composition (Fig 2,

S6), these factors had relatively weak and variable impacts on bacterial community richness (Fig S15A-C; Table S8A). The $\alpha$-diversity of communities (considering both OTU richness and evenness) varied only across host species (Figs S8A-B; see Fig S9 for rarefied open reference communities); Tables S8B and S9); whereas both factors significantly affected the $\beta$ diversity (Figs S8C-D; see Figs S10 and S11 for rarefied open reference communities; Table S8C), indicating significant community turnover across species and sampling location. Interestingly, $\beta$ diversity was higher in dragonflies collected from sites in Southern India (Agumbe, Bangalore, 
497 and Shendurney) compared to North Indian locations (Bordubi and Nagpur) (Figs S8D, S10B).

498 These results show that host-specific and environmental factors together govern bacterial

499 community composition and turnover, with the latter having larger impacts.

500

501

Dragonflies show host-specific dietary specialization

502 To determine whether host-specific bacterial communities reflect host-specific diets, we next 503 tested for dietary specialization across the three best-sampled dragonfly species, O. pruinosum,

504 O. sabina, and P. flavescens collected from Agumbe region. We obtained 12.4\% prey reads, out

505 of a total of 2.1 million reads (Fig S3). Our PERMANOVA analysis revealed that library size did

506 not influence the prey composition in dragonfly guts (Table S2C and D).

507 The pruned prey communities of both Orthetrum species had significantly higher richness

508 compared to $P$. flavescens $(\mathrm{P}<0.01$, Kruskal Wallis' Chi-square $=16.91, \mathrm{df}=2$, post-hoc Conover

509 test: $\mathrm{OP}$ vs. $\mathrm{PF}: \mathrm{P}<0.01$, OS vs. $\mathrm{PF}: \mathrm{P}<0.01$, OP vs $\mathrm{OS}: \mathrm{P}=0.04)$, as well as greater diversity

$510(\mathrm{P}=0.003$, Kruskal Wallis' Chi-squared: $11.41, \mathrm{df}=2$, post-hoc Conover test: OP vs. $\mathrm{PF}: \mathrm{P}=0.04$,

511 OS vs. PF: $\mathrm{P}=0.0008$, OP vs OS: $\mathrm{P}=0.03$ ) (Fig 3A-B; see Fig S16A-B for rarefied mean

512 community). These patterns mirrored the bacterial communities associated with these hosts (Figs

513 3A-B, S16A-B): Orthetrum had higher bacterial diversity (Kruskal Wallis' Chi-squared: 7.39,

$514 \mathrm{df}=2, \mathrm{P}=0.02$, post-hoc Conover test: $\mathrm{OP}$ vs. $\mathrm{PF}: \mathrm{P}=0.03$, OS vs. $\mathrm{PF}: \mathrm{P}=0.04$, OP vs. OS: $\mathrm{P}=0.69$ )

515 (Figs 3A, S16A), though not significantly higher richness (Kruskal Wallis' Chi-squared: 1.99,

$516 \mathrm{df}=2, \mathrm{P}=0.3$ ) (Figs $3 \mathrm{~B}$ and $\mathrm{S} 16 \mathrm{~B}$, though the trend was similar). The correlated differences in

517 prey and gut bacterial communities suggest that they may be causally linked.

518 
519 Notably, we observed striking differences between the diets of the three dragonfly species (Figs

520 3A-B and S17; Table 2A-B). Orthetrum pruinosum and O. sabina had similar diets composed

521 predominantly of Dipterans ( $83 \%$ and $68 \%$ respectively), whereas $P$. flavescens consumed more

522 Odonates ( $88 \%$ of prey OTUs, potentially indicating conspecific predation) (Fig S17). This was

523 also evident in our PERMANOVA results, which revealed that host species was an important

524 factor driving prey composition (Table 2A). Finally, our classification analysis (CAPdiscrim)

525 based on prey composition also categorized significant proportions (75\%) of samples correctly

526 into the respective groups (Table 2B), highlighting host specific dietary patterns in dragonflies.

527 Since all the dragonflies collected for dietary analysis were collected from Agumbe region, we

528 examined the gut bacterial composition of dragonflies (of the three well-sampled species)

529 collected specifically from this area. Concordant to our previous analysis, we found a strong

530 correspondence between the effect of host identity on dietary as well as the bacterial composition

531 of dragonflies (Figs 3C and S16C vs. S18; Table 2 vs. Table S10). Similarly, CAPdiscrim

532 analysis also showed comparable classification success for samples based on both gut bacterial

533 composition (81\%) and prey composition (75\%).

534

535 Overall, these results suggest that (a) O. sabina and O. pruinosum primarily consume small

536 dipterans and lepidopterans (Fig S17); (b) but their target prey community still differed

537 substantially (Fig 3C); and (c) P. flavescens is a specialized predator that predominantly targets

538 other Odonates (Figs 3C and S17). Thus, a host-specific dietary pattern can potentially introduce

539 distinct bacteria into host guts, directly determining the observed host-specific gut bacterial

540 communities of dragonflies. 
542 Dragonfly gut bacterial communities are predominantly neutrally assembled

543 Next, we specifically tested whether dragonfly gut bacterial communities are acquired passively

544 through the diet, with relatively weak host-imposed filters. We estimated the fraction of the

545 bacterial community whose occurrence and abundance across hosts was consistent with neutral

546 vs. non-neutral assembly, analyzing communities from all samples collected from a given

547 location (regardless of host species). We found that a large fraction of bacterial OTUs was

548 predicted to be neutrally assembled (mean $72 \pm 0.08 \%$ with $99 \%$ confidence intervals, Fig

549 S19A-E; mean $63 \pm 0.08 \%$ with $95 \%$ CI; ), i.e., whose distribution across hosts matched

550 expectations from a model simulating assembly via random OTU dispersal. We also compared

551 the fit of the Sloan models with that of binomial models (as suggested by Burns et al., 2016)

552 using both $\mathrm{R}^{2}$ and AIC estimation. In each case, the Sloan model explained more variation in the 553 data than the Binomial model (Table S11). This result suggested that the local communities are

554 not just a random subset of the meta-communities and dispersal plays an important role in

555 structuring the local communities. Beta diversity (variation in composition across local

556 communities) is therefore better explained when we account for dispersal between local

557 communities (i.e. Sloan's model). The proportion of neutrally distributed gut bacteria varied

558 across locations (Figs 4 and S19). Dragonflies collected from Bordubi had the highest proportion

559 of neutrally assembled bacteria (83\%), while those from Nagpur had the lowest proportion of

560 neutrally assembled gut bacteria (63\%; Figs 4 and S19). As expected, we observed the opposite

561 patterns for OTUs whose distribution was consistent with positive or negative selection (Fig 4).

562 We found similar results when we rarefied samples to the lowest read count per sample (e.g.

$56374 \%$ OTUs were predicted to be neutrally assembled, compared to $72 \%$ for the full dataset),

564 suggesting that the model fitting was not sensitive to different read depth across samples. Finally, 
565 pooling all OTUs predicted to be under positive selection (across locations), we found that their

566 taxonomic diversity was either higher than or comparable to OTUs that were neutrally

567 distributed or under purifying/negative selection (Fig S20). This result suggests that it is unlikely

568 that dragonflies impose strong positive selection favoring a specific, shared set of functionally

569 important bacteria. However, it is also possible that any functional traits selected by the host are

570 not phylogenetically conserved (Louca et al., 2018), resulting in positive selection for unrelated

571 bacteria. Currently, we cannot distinguish between these alternatives.

572

573 Since we had relatively low sample sizes for each dragonfly species in a given location, we had

restricted our analysis (above) to pooled samples - i.e., we included data from all dragonflies in a

575 particular sampling location. However, we also attempted to investigate host species-specific

576 patterns of gut bacterial community assembly (Fig S21). We found that host species and

577 sampling location both had a significant impact on the proportion of bacteria that are neutrally

578 assembled (Table S12A and Fig S21A; also see Table S12B and Fig S21B for bacteria under

579 positive selection). The dragonfly P. flavescens (which hunts other Odonates, which are also

580 carnivorous and can also house non-specific neutrally assembled gut bacteria) had a higher

581 proportion of neutrally assembled gut bacteria, in comparison to O. sabina and O. pruinosum

582 (that predates on insect orders which can have host specific gut microbiota).

584 Bacterial cells rarely adhere inside dragonfly guts

585 Finally, to test whether bacterial cells adhere to dragonfly guts or are housed in specialized

586 structures, we dissected the guts of three species (O. sabina, O. pruinosum, and P. flavescens)

587 and probed for bacteria using FISH (Fig 5). Our positive controls (bacterial smears on slides) 
588 showed a strong eubacterial signal, indicative of proper binding (Fig S22 A-B); while our 589 negative control (see methods) did not show a signal. However, we did not find any eubacterial 590 signal in the foregut (Fig 5B-D), indicating that bacteria were either absent or rare in this part of 591 the gut. Since we did not find a signal with the general eubacterial probe, we did not test foregut 592 sections with the Wolbachia-specific probe. In P. flavescens, only the eubacterial probe showed a 593 positive signal inside columnar folds ( 5 of 5 tested individuals; 3 with small patches of bacteria) 594 (Fig 5E and H); whereas Wolbachia was absent (Fig 5K and N), corroborating our amplicon 595 sequencing results. The midgut and hindgut of both Orthetrum species were positive for 596 eubacterial and Wolbachia-specific probes (Fig 5F-P; all 5 tested individuals of each species), 597 although the signal was weak and localized to a small cluster of bacteria in the gaps between 598 columnar cellular folds. Interesting exceptions were observed in two O. sabina individuals where 599 Wolbachia appeared to be sequestered within a specific tissue structure (Fig 5L); the functional 600 significance of this pattern requires further work. Overall, the lack of a clear signal of bacterial 601 gut colonization suggests at best a weak relationship with the host.

602

603

\section{DISCUSSION}

604

605

Host selection is generally considered to be a strong force shaping the gut bacterial communities 606 of animals (Colman, Toolson \& Takacs-Vesbach, 2012; Engel \& Moran, 2013; Yun et al., 2014) 607 and is expected to stabilize communities (Lozupone et al., 2012; Coyte, Schluter \& Foster, 2015; Foster et al., 2017) in the face of environmental variation. Here, we tested this prediction by analyzing host associated gut bacteria across spatially separated populations of six dragonfly

610 species. Our key results contrast multiple findings from prior work: (a) adult dragonfly bacterial 
611 communities are twice as rich and diverse as other carnivorous insects, including Odonates

612 (Jones, Sanchez \& Fierer, 2013; Yun et al., 2014); (b) geographic location explain more variation

613 in bacterial community composition than host species identity; (c) adult dragonflies have

614 specialized diets that reflect patterns of variation in gut bacterial communities, and (d) the adult

615 gut community is predominantly neutrally assembled with regard to host species, showing little

616 evidence of the strong host selection reported for many other insects (Engel \& Moran, 2013; Yun

617 et al., 2014). Thus, our work highlights the importance of analyzing gut microbial communities

618 of natural host populations in the context of natural variation in geography and host taxonomy.

619

620 Our findings corroborate previous studies showing a significant geographical structure in the

621 microbiomes of well-studied animal species such as humans, flies, and bees (Corby-Harris et al.,

622 2007; Turnbaugh et al., 2009; Costello et al., 2009). Our prior analysis of culturable gut bacteria

623 in dragonflies had also shown a weak yet significant effect of sampling location on the gut

624 bacterial community (Nair \& Agashe, 2016). In this study, the presence of a strong geographic

625 structure (Fig 2B) despite incorporating long-distance migrants like Pantala (Hobson et al.,

626 2012) further highlights the importance of environmental factors shaping the gut bacterial

627 community composition. However, the dragonfly bacterial community structure is not strongly

628 associated with the distance between sites (Figs 1A, S8, and S10). For instance, dragonflies of

629 the same species collected from relatively close sites - Bangalore, Agumbe, and Shendurney -

630 had distinct gut bacterial community composition, suggesting that a combination of multiple

631 locally-acting factors may drive the composition of site-specific gut bacterial communities (Figs

632 1A, 2B, S8, and S10). These factors may include specific environmental conditions (e.g.,

633 temperature, precipitation, and soil $\mathrm{pH}$ ) that drive variation in environmental microbes; site- 
634 specific variation in host imposed selection acting on similar environmental microbes; variation

635 in insect prey communities driving differential dispersal into host guts; or local host diet

636 specialization (Dillon \& Dillon, 2004; Osei-Poku et al., 2012; Engel \& Moran, 2013).

637 Dragonflies are known to be generalist predators of various insects (Corbet, 2004; Olberg et al.,

638 2005; Kaunisto et al., 2017), which often house host-specific microbiota (Dillon \& Dillon, 2004;

639 Engel \& Moran, 2013; Yun et al., 2014). Hence, a shift in the prey base due to geographical

640 variation may directly or indirectly contribute to changes in the gut bacterial community of

641 predatory dragonflies. We do acknowledge that among different environmental factors, seasonal

642 variation can play a crucial role in gut bacterial community composition. However owing to our

643 limited sampling effort we could not address the effect of seasonality in our present study, which

644 remains to be tested in future studies.

645

646 Despite the major impacts of geographic location, concordant to our previous study (Nair \&

647 Agashe, 2016) we found that adults of each dragonfly host genus house a distinct gut bacterial

648 community. What explains this partial host-specificity in the dragonfly gut microbiome? Our

649 results suggest a potential role for phylogenetically conserved host level processes in shaping the

650 gut community. For instance, both species from the genus Orthetrum shared a significant

651 proportion of their gut community, whereas dragonflies from the genus Urothemis - the

652 phylogenetically most distinct genus in our dataset (Ware, May \& Kjer, 2007) - had very

653 different gut bacteria. As is known for host taxonomy, host phylogeny could potentially structure

654 insect gut microbiota through active or passive filters imposed by host morphology, physiology,

655 development, immune function, social interactions or diet (Dillon \& Dillon, 2004; Sullam et al.,

656 2012; Colman, Toolson \& Takacs-Vesbach, 2012; Jones, Sanchez \& Fierer, 2013; Engel \& 
657 Moran, 2013; Aksoy et al., 2014; Yun et al., 2014; Moran \& Sloan, 2015); though this remains to 658 be tested.

659

660 Broadly speaking, host-specific gut microbiota may reflect host specific diets and/or host 661 specific selective filters (Colman, Toolson \& Takacs-Vesbach, 2012; Engel \& Moran, 2013).

662 Unfortunately, information on dragonfly diet is scarce because of their rapid and unpredictable 663 movements that make field observations difficult (Corbet, 2004). However, limited behavioral 664 observations in natural and enclosed populations suggest that dragonflies are generalists (Fraser, 665 1933; Corbet, 2004; Stoks \& Córdoba-Aguilar, 2012). A recent analysis of prey DNA from the 666 faeces of three odonate species in Finland (including dragonflies and damselflies) found large 667 dietary overlaps (Kaunisto et al. (2017), supporting the idea that odonates are generalists. In 668 contrast, using a similar approach, we found that three common, sympatric dragonflies in India 669 (O. sabina, O. pruinosum, and P. flavescens) consume very distinct insect communities. Our 670 result is supported by prior behavioural observations (Fraser, 1933; Corbet, 2004) and our own 671 observations of Orthetrum spp. preying on flies, and mosquitoes and $P$. flavescens consuming 672 other dragonflies (personal observation of Orthetrum spp. and P. flavescens foraging, by 673 Shantanu Joshi and Rittik Deb, year 2015-2016). These dietary differences were also strongly 674 reflected in the diversity and richness of gut bacteria, suggesting a direct association between 675 dietary and gut bacterial diversity. Our results suggest that each dragonfly species may have a 676 unique dietary niche that acts as a passive filter modulating the entry of environmental 677 microbiota into the gut.

678 However, we caution that our results indicate correlation rather than direct causation, and further 679 work is necessary to assign causality. 
681 Although this hypothesis requires further validation, we suggest that such dietary specialization 682 rather than strong host selection - is the primary driver of variation in dragonfly gut bacterial 683 communities. Indeed, simulations using Sloan's neutral assembly model (2006) revealed that 684 bacterial communities were predominantly neutrally assembled with regard to host species and 685 that assembly varied across geographic location and host species. Two additional lines of 686 evidence support our conclusion that adult dragonfly gut bacterial communities are primarily 687 structured via passive processes. First, the high taxonomic diversity of "selected" bacterial OTUs 688 could suggest a lack of selection for a specific set of phylogenetically conserved bacterial 689 functional traits (but see Louca et al. (2018)). Second, our FISH analysis also indicated a weak 690 association with hosts. The rare observations of Wolbachia cells inside globular sacks in $O$. 691 sabina deserve further attention as a possible special case of strong dragonfly-bacterial 692 interactions. Nonetheless, our results indicate that although microbiota are transient, with likely 693 limited impacts on the dragonfly host (comparable to observations in butterflies (Hammer et al., 694 2017; Phalnikar, Kunte \& Agashe, 2018)), the microbiome may be largely shaped by host diet 695 via its impact on microbial dispersal, nutrient availability and colonization .

697 Conclusions

698 Our analysis of the patterns of spatial and host-specific variation in the diet and gut bacterial 699 communities of multiple wild-collected adult dragonflies highlights two key points. First, we 700 suggest that environmental factors that may alter bacterial community stability should be given 701 more importance when drawing general conclusions about host-microbe interactions. Second, 702 while explaining variation in microbial community composition, it is important to explicitly 
703 consider neutral processes along with selection. The lack of significant host-microbiome

704 relationships in dragonfly adults may arise from multiple reasons that remain to be tested. For

705 instance, microbial mutualists may offer no benefit if dragonflies have endogenous digestive

706 enzymes or acquire all essential nutrients from their prey. Alternatively, microbes may not be

707 able to colonize the dragonfly gut because of the host's variable and omnivorous diet, or for

708 reasons of historical chance. We hope that our work encourages further analysis of variation in

709 gut microbiomes of natural insect populations, as well as experimental tests of the role of neutral

710 vs. selective processes in the assembly of host-associated microbial communities.

711

712 DATA ACCESSIBILITY

713 All data are made available in public repositories. Sequencing data and metadata have been

714 uploaded on the European Nucleotide Archive website (PRJEB32318, PRJEB32316,

715 PRJEB32311, PRJEB32309 for gut bacterial data, PRJEB32308 for diet data). OTU tables are

716 made available at Figshare (gut bacteria raw data file without any pruning:

717 10.6084/m9.figshare.9632084, diet raw data file without any pruning:

718 10.6084/m9.figshare.9632096)

719

720

ACKNOWLEDGEMENTS

721 We thank the editor and the two anonymous reviewers for their detailed and insightful

722 comments. We thank members of the Agashe lab for critically reading the manuscript. We thank

723 Krushnamegh Kunte for contributing samples from Shendurney Wildlife Sanctuary; Agumbe

724 Rainforest Research Station, Rohini Balakrishnan, and Sarita and Ramanuj Dasgupta for

725 logistical support; Ashish Tiple, Krushnamegh Kunte, Kruttika Phalnikar, Manjunatha Reddy,

726 Ronita Mukherjee, Sudhakar Gowda and Saira Guha for field assistance; Shantanu Joshi for help 
727 with identifying dragonflies; and Kruttika Phalnikar for help with QIIME analysis. We

728 acknowledge funding and support from the National Centre for Biological Sciences the

729 Department of Science and Technology, India (INSPIRE Faculty award IFA-13 LSBM-64 to

730 Deepa Agashe), and a Wellcome Trust/DBT India Alliance Fellowship (grant number

731 IA/I/17/1/503091 to Deepa Agashe).

732

733 AUTHOR CONTRIBUTIONS

734 RD: analyzed Miseq data; designed work, collected and prepared samples, and analyzed data for 735 diet, FISH and qPCR experiments; carried out community assembly analysis; prepared figures 736 and drafted the manuscript. AN: collected and prepared samples for gut bacterial analysis. DA:

737 conceived the study; designed experiments; collected samples; acquired funding; wrote the 738 manuscript.

739

740 CONFLICT OF INTEREST

741 Authors declare no conflict of interests. 


\section{BIBLIOGRAPHY}

Adair KL, Wilson M, Bost A, Douglas AE. 2018. Microbial community assembly in wild populations of the fruit fly Drosophila melanogaster. The ISME Journal 12:959-972.

Aksoy E, Telleria EL, Echodu R, Wu Y, Okedi LM, Weiss BL, Aksoy S, Caccone A. 2014. Analysis of multiple tsetse fly populations in Uganda reveals limited diversity and species-specific gut microbiota. Applied and Environmental Microbiology 80:4301-4312.

Anderson MJ, Willis TJ. 2003. Canonical Analysis ofPrincipal Coordinates: A Useful Method of Constrained Ordination for Ecology. Ecology 84:511-525.

Antwis RE, Griffiths SM, Harrison XA, Aranega-Bou P, Arce A, Bettridge AS, Brailsford FL, de Menezes A, Devaynes A, Forbes KM, Fry EL, Goodhead I, Haskell E, Heys C, James C, Johnston SR, Lewis GR, Lewis Z, Macey MC, McCarthy A, McDonald JE, MejiaFlorez NL, O'Brien D, Orland C, Pautasso M, Reid WDK, Robinson HA, Wilson K, Sutherland WJ. 2017. Fifty important research questions in microbial ecology. FEMS Microbiology Ecology 93.

Babraham Bioinformatics - FastQC A Quality Control tool for High Throughput Sequence Data

De Barba M, Miquel C, Boyer F, Mercier C, Rioux D, Coissac E, Taberlet P. 2014. DNA metabarcoding multiplexing and validation of data accuracy for diet assessment: application to omnivorous diet. Molecular ecology resources 14:306-323.

Barrow PA, Brooker BE, Fuller R, Newport MJ. 1980. The attachment of bacteria to the gastric epithelium of the pig and its importance in the microecology of the intestine. The Journal of applied bacteriology 48:147-154.

Bokulich NA, Subramanian S, Faith JJ, Gevers D, Gordon JI, Knight R, Mills DA, Caporaso JG. 2013. Quality-filtering vastly improves diversity estimates from Illumina amplicon sequencing. Nature Methods 10:57-59.

bold: Interface to Bold Systems API version 0.9.0 from CRAN

Broderick NA, Lemaitre B. 2012. Gut-associated microbes of Drosophila melanogaster. Gut microbes 3:307-321.

Burns AR, Stephens WZ, Stagaman K, Wong S, Rawls JF, Guillemin K, Bohannan BJ. 2016. Contribution of neutral processes to the assembly of gut microbial communities in the zebrafish over host development. The ISME Journal 10:655-664.

Caporaso JG, Kuczynski J, Stombaugh J, Bittinger K, Bushman FD, Costello EK, Fierer N, Peña AG, Goodrich JK, Gordon JI, Huttley GA, Kelley ST, Knights D, Koenig JE, Ley RE, Lozupone CA, McDonald D, Muegge BD, Pirrung M, Reeder J, Sevinsky JR, Turnbaugh PJ, Walters WA, Widmann J, Yatsunenko T, Zaneveld J, Knight R. 2010. QIIME allows analysis of high-throughput community sequencing data. Nature Methods 7:335-336. 
778

779

780

781

782

783

784

785

786

787

788

789

790

791

792

793

794

795

796

797

798

799

800

801

802

803

804

805

806

807

808

809

810

811

812

Charroux B, Royet J. 2012. Gut-microbiota interactions in non-mammals: what can we learn from Drosophila? Seminars in Immunology 24:17-24.

Chow S, Suzuki S, Matsunaga T, Lavery S, Jeffs A, Takeyama H. 2011. Investigation on natural diets of larval marine animals using peptide nucleic acid-directed polymerase chain reaction clamping. Marine Biotechnology 13:305-313.

Clarke KR, Warwick RM. 1998. A taxonomic distinctness index and its statistical properties. Journal of Applied Ecology 35:523-531.

Le Clec'h W, Chevalier FD, Genty L, Bertaux J, Bouchon D, Sicard M. 2013. Cannibalism and predation as paths for horizontal passage of Wolbachia between terrestrial isopods. Plos One 8:e60232.

Colman DR, Toolson EC, Takacs-Vesbach CD. 2012. Do diet and taxonomy influence insect gut bacterial communities? Molecular Ecology 21:5124-5137.

Corbet PS. 2004. Dragonflies: behaviour and ecology of Odonata. Harley Books.

Corby-Harris V, Pontaroli AC, Shimkets LJ, Bennetzen JL, Habel KE, Promislow DEL. 2007. Geographical distribution and diversity of bacteria associated with natural populations of Drosophila melanogaster. Applied and Environmental Microbiology 73:3470-3479.

Costello EK, Lauber CL, Hamady M, Fierer N, Gordon JI, Knight R. 2009. Bacterial community variation in human body habitats across space and time. Science 326:1694-1697.

Costello EK, Stagaman K, Dethlefsen L, Bohannan BJM, Relman DA. 2012. The application of ecological theory toward an understanding of the human microbiome. Science 336:12551262.

Coyte KZ, Schluter J, Foster KR. 2015. The ecology of the microbiome: Networks, competition, and stability. Science 350:663-666.

CRAN - Package PMCMR

Deagle BE, Jarman SN, Coissac E, Pompanon F, Taberlet P. 2014. DNA metabarcoding and the cytochrome c oxidase subunit I marker: not a perfect match. Biology Letters 10.

DeSantis TZ, Hugenholtz P, Larsen N, Rojas M, Brodie EL, Keller K, Huber T, Dalevi D, Hu P, Andersen GL. 2006. Greengenes, a chimera-checked 16S rRNA gene database and workbench compatible with ARB. Applied and Environmental Microbiology 72:50695072.

Dijkstra KDB, Kalkman VJ, Dow RA, Stokvis FR, Vantol J. 2014. Redefining the damselfly families: a comprehensive molecular phylogeny of Zygoptera (Odonata). Systematic entomology 39:68-96.

Dillon RJ, Dillon VM. 2004. The gut bacteria of insects: nonpathogenic interactions. Annual Review of Entomology 49:71-92. 
813 Dixon P. 2003. VEGAN, a package of R functions for community ecology. Journal of vegetation

814

815

816

817

818

819

820

821

822

823

824

825

826

827

828

829

830

831

832

833

834

835

836

837

838

839

840

841

842

843

844

845

846

847 science : official organ of the International Association for Vegetation Science 14:927.

Edgar RC. 2010. Search and clustering orders of magnitude faster than BLAST. Bioinformatics 26:2460-2461.

Elzhov TV, Mullen KM, Spiess A, Bolker B. 2010. R interface to the Levenberg-Marquardt nonlinear least-squares algorithm found in MINPACK. Plus Support for Bounds.

Engel P, Moran NA. 2013. The gut microbiota of insects - diversity in structure and function. FEMS Microbiology Reviews 37:699-735.

Fierer N, Bradford MA, Jackson RB. 2007. Toward an ecological classification of soil bacteria. Ecology 88:1354-1364.

De Filippo C, Cavalieri D, Di Paola M, Ramazzotti M, Poullet JB, Massart S, Collini S, Pieraccini G, Lionetti P. 2010. Impact of diet in shaping gut microbiota revealed by a comparative study in children from Europe and rural Africa. Proceedings of the National Academy of Sciences of the United States of America 107:14691-14696.

Foster KR, Schluter J, Coyte KZ, Rakoff-Nahoum S. 2017. The evolution of the host microbiome as an ecosystem on a leash. Nature 548:43-51.

Fraser FC. 1933. The Fauna of British India, Including Ceylon and Burma: Arthropoda: Odonata. Taylor and Francis of London: British government in India.

Fuller R, Turvey A. 1971. [Bacteria associated with the intestinal wall of the fowl (Gallus domesticus)]. The Journal of applied bacteriology 34:617-622.

Haas BJ, Gevers D, Earl AM, Feldgarden M, Ward DV, Giannoukos G, Ciulla D, Tabbaa D, Highlander SK, Sodergren E, Methé B, DeSantis TZ, Human Microbiome Consortium, Petrosino JF, Knight R, Birren BW. 2011. Chimeric 16S rRNA sequence formation and detection in Sanger and 454-pyrosequenced PCR amplicons. Genome Research 21:494-504.

Hammer TJ, Janzen DH, Hallwachs W, Jaffe SP, Fierer N. 2017. Caterpillars lack a resident gut microbiome. Proceedings of the National Academy of Sciences of the United States of America 114:9641-9646.

Hebert PDN, Ratnasingham S, deWaard JR. 2003. Barcoding animal life: cytochrome c oxidase subunit 1 divergences among closely related species. Proceedings. Biological Sciences / the Royal Society 270 Suppl 1:S96-9.

Heddi A, Grenier AM, Khatchadourian C, Charles H, Nardon P. 1999. Four intracellular genomes direct weevil biology: nuclear, mitochondrial, principal endosymbiont, and Wolbachia. Proceedings of the National Academy of Sciences of the United States of America 96:6814-6819. 
848

849

850

851

852

853

854

855

856

857

858

859

860

861

862

863

864

865

866

867

868

869

870

871

872

873

874

875

876

877

878

879

880

881

882

Hobson KA, Anderson RC, Soto DX, Wassenaar LI. 2012. Isotopic evidence that dragonflies (Pantala flavescens) migrating through the Maldives come from the northern Indian subcontinent. Plos One 7:e52594.

Hubbell SP. 2001. The Unified Neutral Theory of Biodiversity and Biogeography. USA: Princeton University Press.

Huse SM, Welch DM, Morrison HG, Sogin ML. 2010. Ironing out the wrinkles in the rare biosphere through improved OTU clustering. Environmental Microbiology 12:18891898.

Jones RT, Sanchez LG, Fierer N. 2013. A cross-taxon analysis of insect-associated bacterial diversity. Plos One 8:e61218.

Joshi S. 2018. History of species pages. Odonata of India, v. 1.10. Indian Foundation for Butterflies.

Jr FH, Dupont C. 2008. Hmisc: harrell miscellaneous. $R$ package version.

Kamenova S, Mayer R, Coissac E, Plantegenest M, Traugott M. 2017. Comparing three types of dietary samples for prey DNA decay in an insect generalist predator. BioRxiv.

Kaunisto KM, Roslin T, Sääksjärvi IE, Vesterinen EJ. 2017. Pellets of proof: First glimpse of the dietary composition of adult odonates as revealed by metabarcoding of feces. Ecology and evolution 7:8588-8598.

Kikuchi Y, Hosokawa T, Fukatsu T. 2007. Insect-microbe mutualism without vertical transmission: a stinkbug acquires a beneficial gut symbiont from the environment every generation. Applied and Environmental Microbiology 73:4308-4316.

Kindt R, Kindt MR. 2017. Package' ' 'BiodiversityR.

Koleff P, Gaston KJ, Lennon JJ. 2003. Measuring beta diversity for presence-absence data. Journal of Animal Ecology 72:367-382.

Kostic AD, Howitt MR, Garrett WS. 2013. Exploring host-microbiota interactions in animal models and humans. Genes \& Development 27:701-718.

Kuhn M. 2008. Caret package. Journal of statistical software 28:1-26.

Engel P, James RR, Koga R, Kwong WK, McFrederick QS, Moran NA. 2013. Standard methods for research on Apis mellifera gut symbionts. Journal of Apicultural Research.

Langille MGI, Zaneveld J, Caporaso JG, McDonald D, Knights D, Reyes JA, Clemente JC, Burkepile DE, Vega Thurber RL, Knight R, Beiko RG, Huttenhower C. 2013. Predictive functional profiling of microbial communities using 16S rRNA marker gene sequences. Nature Biotechnology 31:814-821.

Ley RE, Peterson DA, Gordon JI. 2006. Ecological and evolutionary forces shaping microbial diversity in the human intestine. Cell 124:837-848. 
883

884

885

886

887

888

889

890

891

892

893

894

895

896

897

898

899

900

901

902

903

904

905

906

907

908

909

910

911

912

913

914

915

916

917

918

Louca S, Polz MF, Mazel F, Albright MBN, Huber JA, O’Connor MI, Ackermann M, Hahn AS, Srivastava DS, Crowe SA, Doebeli M, Parfrey LW. 2018. Function and functional redundancy in microbial systems. Nature ecology \& evolution 2:936-943.

Lozupone CA, Stombaugh JI, Gordon JI, Jansson JK, Knight R. 2012. Diversity, stability and resilience of the human gut microbiota. Nature 489:220-230.

Martinson VG, Douglas AE, Jaenike J. 2017. Community structure of the gut microbiota in sympatric species of wild Drosophila. Ecology Letters 20:629-639.

McFall-Ngai M, Hadfield MG, Bosch TCG, Carey HV, Domazet-Lošo T, Douglas AE, Dubilier N, Eberl G, Fukami T, Gilbert SF, Hentschel U, King N, Kjelleberg S, Knoll AH, Kremer N, Mazmanian SK, Metcalf JL, Nealson K, Pierce NE, Rawls JF, Reid A, Ruby EG, Rumpho M, Sanders JG, Tautz D, Wernegreen JJ. 2013. Animals in a bacterial world, a new imperative for the life sciences. Proceedings of the National Academy of Sciences of the United States of America 110:3229-3236.

McMurdie PJ, Holmes S. 2014. Waste not, want not: why rarefying microbiome data is inadmissible. PLoS Computational Biology 10:e1003531.

Moran NA, Sloan DB. 2015. The hologenome concept: helpful or hollow? PLoS Biology 13:e1002311.

Morrow JL, Frommer M, Shearman DCA, Riegler M. 2015. The Microbiome of Field-Caught and Laboratory-Adapted Australian Tephritid Fruit Fly Species with Different Host Plant Use and Specialisation. Microbial Ecology 70:498-508.

Murtagh F, Legendre P. 2014. Ward's Hierarchical Agglomerative Clustering Method: Which Algorithms Implement Ward's Criterion? Journal of Classification 31:274-295.

Nair A, Agashe D. 2016. Host-specific spatial and temporal variation in culturable gut bacterial communities of dragonflies. Current science 110:1513-1523.

Nicholson JK, Holmes E, Kinross J, Burcelin R, Gibson G, Jia W, Pettersson S. 2012. Host-gut microbiota metabolic interactions. Science 336:1262-1267.

Oksanen J, Blanchet FG, Friendly M, Kindt R, Legendre P, McGlinn D, Minchin P, O'Hara R, Simpson G, Solymos P, Henry M, Stevens H, Szoecs E, Wagner H. 2017. Vegan: Community Ecology Package. R Package Version 2.4-4.

Olberg RM, Worthington AH, Fox JL, Bessette CE, Loosemore MP. 2005. Prey size selection and distance estimation in foraging adult dragonflies. Journal of Comparative Physiology. A, Neuroethology, Sensory, Neural, and Behavioral Physiology 191:791797.

Osei-Poku J, Mbogo CM, Palmer WJ, Jiggins FM. 2012. Deep sequencing reveals extensive variation in the gut microbiota of wild mosquitoes from Kenya. Molecular Ecology 21:5138-5150. 
919 Phalnikar K, Kunte K, Agashe D. 2018. Dietary and developmental shifts in butterfly-associated

920

921

922

923

924

925

926

927

928

929

930

931

932

933

934

935

936

937

938

939

940

941

942

943

944

945

946

947

948

949

950

951

952

953 bacterial communities. Royal Society open science 5:171559.

Piñol J, San Andrés V, Clare EL, Mir G, Symondson WOC. 2014. A pragmatic approach to the analysis of diets of generalist predators: the use of next-generation sequencing with no blocking probes. Molecular ecology resources 14:18-26.

Quigley EMM. 2013. Gut bacteria in health and disease. Gastroenterology \& hepatology 9:560569.

R Core Team. 2013. R: A language and environment for statistical computing. Vienna, Austria: R Foundation for Statistical Computing.

Ratnasingham S, Hebert PDN. 2007. bold: The Barcode of Life Data System (http://www.barcodinglife.org). Molecular ecology notes 7:355-364.

Rosindell J, Hubbell SP, Etienne RS. 2011. The unified neutral theory of biodiversity and biogeography at age ten. Trends in Ecology \& Evolution 26:340-348.

Sanders JG, Lukasik P, Frederickson ME, Russell JA, Koga R, Knight R, Pierce NE. 2017. Dramatic Differences in Gut Bacterial Densities Correlate with Diet and Habitat in Rainforest Ants. Integrative and Comparative Biology 57:705-722.

Shukla SP, Sanders JG, Byrne MJ, Pierce NE. 2016. Gut microbiota of dung beetles correspond to dietary specializations of adults and larvae. Molecular Ecology 25:6092-6106.

Da Silva S, Robbe-Masselot C, Raymond A, Mercade-Loubière M, Salvador-Cartier C, Ringot B, Léonard R, Fourquaux I, Ait-Belgnaoui A, Loubière P, Théodorou V, Mercier-Bonin M. 2015. Spatial Localization and Binding of the Probiotic Lactobacillus farciminis to the Rat Intestinal Mucosa: Influence of Chronic Stress. Plos One 10:e0136048.

Sloan WT, Lunn M, Woodcock S, Head IM, Nee S, Curtis TP. 2006. Quantifying the roles of immigration and chance in shaping prokaryote community structure. Environmental Microbiology 8:732-740.

Sloan WT, Woodcock S, Lunn M, Head IM, Curtis TP. 2007. Modeling taxa-abundance distributions in microbial communities using environmental sequence data. Microbial Ecology 53:443-455.

Spor A, Koren O, Ley R. 2011. Unravelling the effects of the environment and host genotype on the gut microbiome. Nature Reviews. Microbiology 9:279-290.

Stoks R, Córdoba-Aguilar A. 2012. Evolutionary ecology of Odonata: a complex life cycle perspective. Annual Review of Entomology 57:249-265.

Sullam KE, Essinger SD, Lozupone CA, O’Connor MP, Rosen GL, Knight R, Kilham SS, Russell JA. 2012. Environmental and ecological factors that shape the gut bacterial communities of fish: a meta-analysis. Molecular Ecology 21:3363-3378. 
954 Terahara T, Chow S, Kurogi H, Lee S-H, Tsukamoto K, Mochioka N, Tanaka H, Takeyama H.

955

956

957

958

959

960

961

962

963

964

965

966

967

968

969

970

971

972

973

974

975

976

977 2011. Efficiency of peptide nucleic acid-directed PCR clamping and its application in the investigation of natural diets of the Japanese eel leptocephali. Plos One 6:e25715.

Turnbaugh PJ, Ridaura VK, Faith JJ, Rey FE, Knight R, Gordon JI. 2009. The effect of diet on the human gut microbiome: a metagenomic analysis in humanized gnotobiotic mice. Science Translational Medicine 1:6ra14.

Vestheim H, Jarman SN. 2008. Blocking primers to enhance PCR amplification of rare sequences in mixed samples - a case study on prey DNA in Antarctic krill stomachs. Frontiers in zoology 5:12.

Ware J, May M, Kjer K. 2007. Phylogeny of the higher Libelluloidea (Anisoptera: Odonata): an exploration of the most speciose superfamily of dragonflies. Molecular Phylogenetics and Evolution 45:289-310.

Woodcock S, van der Gast CJ, Bell T, Lunn M, Curtis TP, Head IM, Sloan WT. 2007. Neutral assembly of bacterial communities. FEMS Microbiology Ecology 62:171-180.

Yadav KK, Bora A, Datta S, Chandel K, Gogoi HK, Prasad GBKS, Veer V. 2015. Molecular characterization of midgut microbiota of Aedes albopictus and Aedes aegypti from Arunachal Pradesh, India. Parasites \& vectors 8:641.

Yun J-H, Roh SW, Whon TW, Jung M-J, Kim M-S, Park D-S, Yoon C, Nam Y-D, Kim Y-J, Choi J-H, Kim JY, Shin NR, Kim SH, Lee WJ, Bae JW. 2014. Insect gut bacterial diversity determined by environmental habitat, diet, developmental stage, and phylogeny of host. Applied and Environmental Microbiology 80:5254-5264.

Zeale MRK, Butlin RK, Barker GLA, Lees DC, Jones G. 2011. Taxon-specific PCR for DNA barcoding arthropod prey in bat faeces. Molecular ecology resources 11:236-244. 
979

980 Figure 1: (A) Map of India showing dragonfly sampling locations. (Map of India: Anuradha 981 Joglekar and Krushnamegh Kunte, NCBS (copyright), see supplemental file 2 for permission).

982 Sampling details are given in Table S1. (B) Orthetrum pruinosum. (C) Othetrum sabina, (D)

983 Pantala flavescens, (E) Trithemis aurora, (F) Urothemis signata, (G) Zyonyx iris. (Picture

984 courtesy: B-F: Dattaprasad Sawant (copyright, see supplemental file 3 for permission), G:

985 Krushnamegh Kunte, NCBS (copyright, see supplemental file 2 for permission). (H) Major

986 bacterial phyla and classes in the dominant gut bacterial communities of sampled dragonflies.

987 Numbers in parentheses indicate the number of OTUs (dominant community) in each taxonomic

988 group. (I) Heat map showing dominant bacterial OTUs across all dragonflies. Each column

989 indicates a host individual (sorted by species), and rows indicate dominant bacterial OTUs

990 clustered based on their abundance across hosts.

991

992 Figure 2: Linear discriminant (LD) plots showing two dominant linear discriminants (LD) that 993 group dragonfly samples based on their gut bacterial community composition (based on Bray994 Curtis distance and open reference OTU picking). Percentage of variance explained by each LD is 995 indicated in parentheses. Each point represents a host individual. Ellipsoids represent 95\% confidence intervals around each group mean, calculated from LD values. Clustering of dragonfly samples based on (A) host species identity (3 well-sampled species) (Table S1A), (B) sampling Shendurney (Table S1), based on their gut bacterial composition. 
1001 Figure 3: Boxplots show (A) Shannon diversity and (B) OTU richness of bacterial and prey 1002 communities of three dragonfly species. Asterisks indicate significant differences in richness 1003 (Kruskal Wallis test). (C) Clustering of dragonfly samples based on dietary composition using LD 1004 analysis, as described in Fig 2.

1005

1006

Figure 4. Barplots show the proportion of bacteria whose distribution is consistent with positive 1007 selection, neutral assembly, or negative/purifying selection, for dragonflies sampled from a given 1008 location.

1009

1010

Figure 5: Examples of Fluorescent in situ hybridisation (FISH) images of dragonfly gut sections using bacteria-specific probes. Host cell nuclei are stained purple with DAPI, eubacteria are green, 1012 and Wolbachia is pink. Arrows highlight bacteria in each section. (A) Representative brightfield image of $P$. flavescens midgut section showing columnar cellular folds covering the gut lumen, 1014 and food particles in the lumen. (B-D) Foregut sections of P. flavescens, $O$. sabina and $O$. pruinosum. Note the lack of eubacterial or Wolbachia signal. (E-G) Midgut and (H-J) hindgut sections of each species, stained with a eubacterial probe. Note the strong eubacterial signal near the columnar folds of $P$. flavescens. (K-M) Midgut and (N-P) hindgut sections of each species, 1018 stained with a Wolbachia-specific probe. Note the lack of signal in P.flavescens, a weak signal in 1019 O. pruinosum, and a large globular structure with Wolbachia in O. sabina. 


\section{Figure 1}

Figure 1

(A) Map of India showing dragonfly sampling locations (Map of India: () Anuradha Joglekar and Krushnamegh Kunte, NCBS). Sampling details are given in Table S1. (B) Orthetrum pruinosum. (C) Othetrum sabina, (D) Pantala flavescens, (E) Trithemis aurora, (F) Urothemis signata, (G) Zyonyx iris. (Photo credit: B-F: @ Dattaprasad Sawant, G: ๑ Krushnamegh Kunte, NCBS. (H) Major bacterial phyla and classes in the dominant gut bacterial communities of sampled dragonflies. Numbers in parentheses indicate the number of OTUs (dominant community) in each taxonomic group. (I) Heat map showing dominant bacterial OTUs across all dragonflies. Each column indicates a host individual (sorted by species), and rows indicate dominant bacterial OTUs clustered based on their abundance across hosts. 
A.

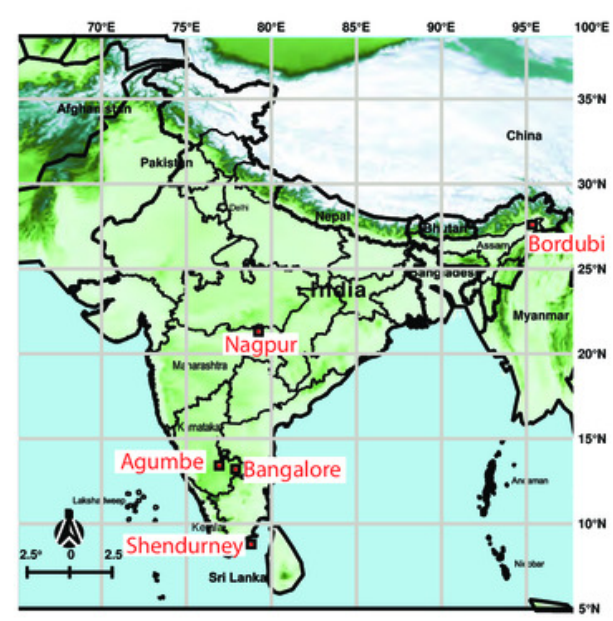

I.
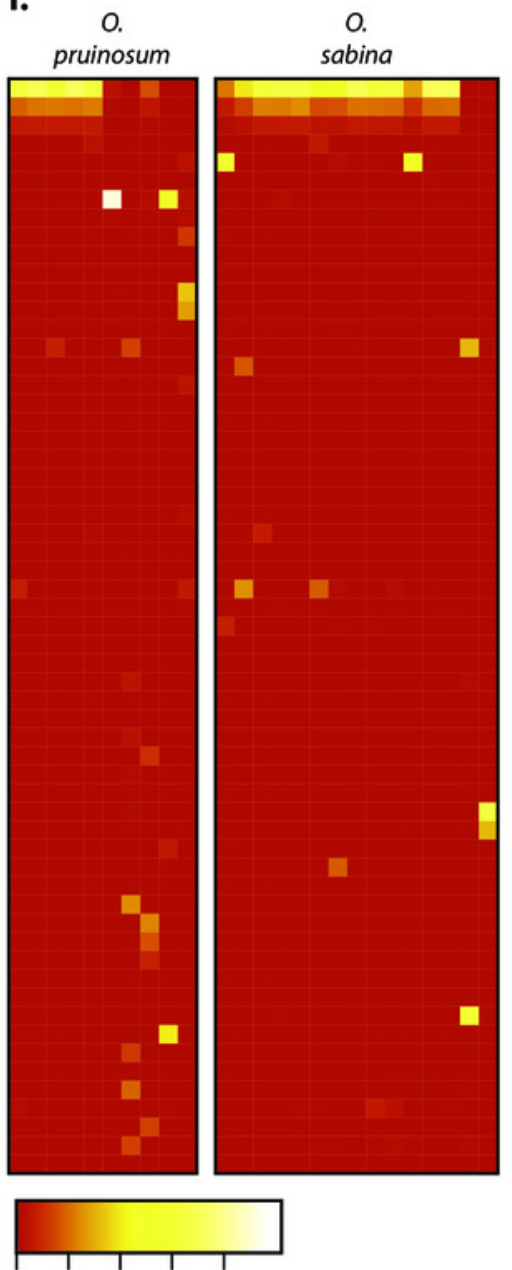

$\begin{array}{llllll}0 & 0.2 & 0.4 & 0.6 & 0.8 & \text { Relative abundance }\end{array}$
B.

Orthetrum pruinosum

C.

Orthetrum sabina

D.

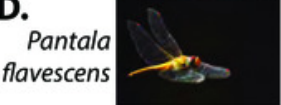

E. Trithemis aurora

F. Urothemis signata

G.

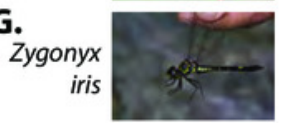

H.

Flavobacteria (3)

$0.4 \%$

(Phylum: Bacteroidetes)

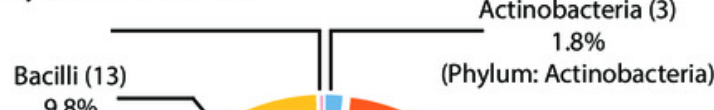

(Phylum: Firmicutes)

\section{CLASS}

Gammaproteobacteria (27)

$39.4 \%$

(Phylum: Proteobacteria)
T. U. Z.

flavescens aurora signata iris

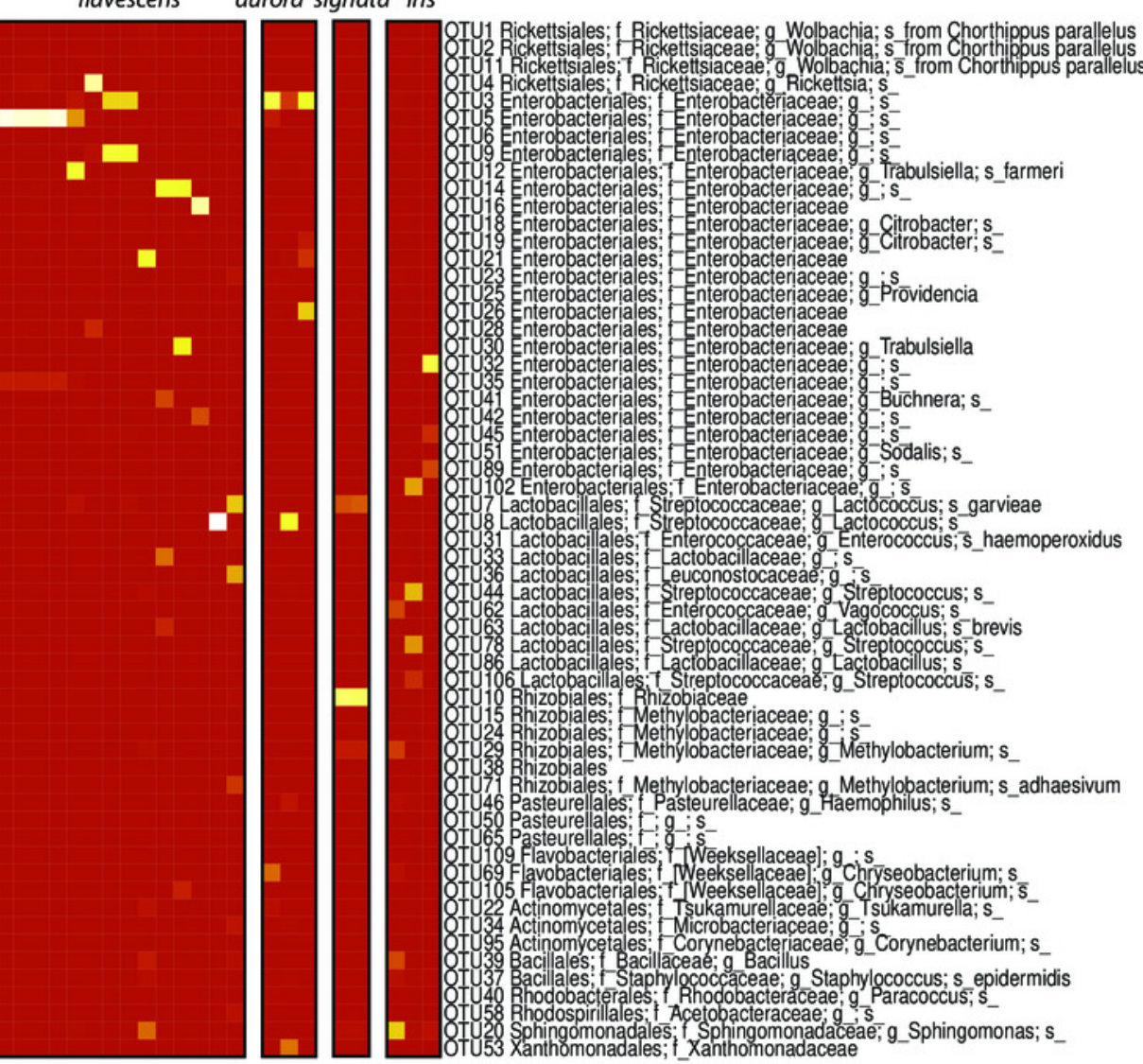




\section{Figure 2}

Figure 2

Figure 2: Linear discriminant (LD) plots showing two dominant linear discriminants (LD) that group dragonfly samples based on their gut bacterial community composition (based on Bray-Curtis distance and open reference OTU picking). Percentage of variance explained by each LD is indicated in parentheses. Each point represents a host individual. Ellipsoids represent 95\% confidence intervals around each group mean, calculated from LD values. Clustering of dragonfly samples based on (A) host species identity ( 3 well-sampled species) (Table S1A), (B) sampling location (for 3 well-sampled species) (Table S1A). (C) Clustering of 5 dragonfly species from Shendurney (Table S1), based on their gut bacterial composition.
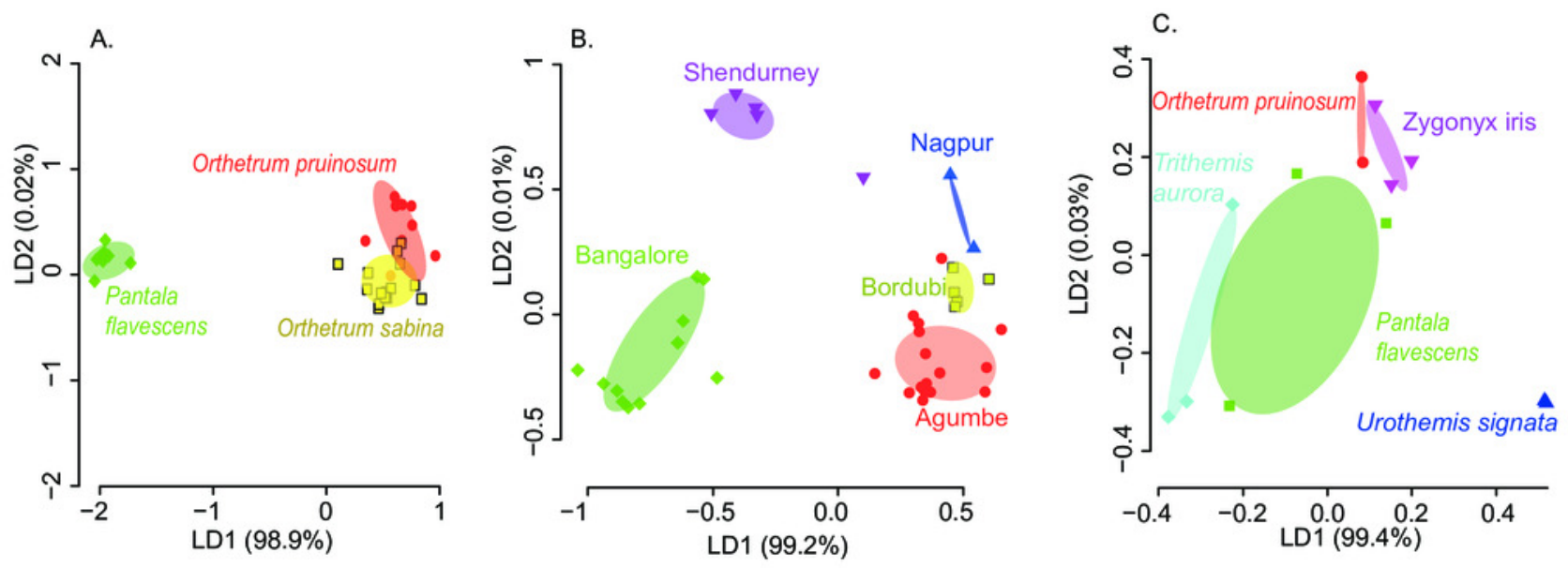


\section{Figure 3}

Figure 3

Boxplots show (A) Shannon diversity and (B) OTU richness of bacterial and prey communities of three dragonfly species. Asterisks indicate significant differences in richness (Kruskal Wallis test). (C) Clustering of dragonfly samples based on dietary composition using LD analysis, as described in Fig 2. 

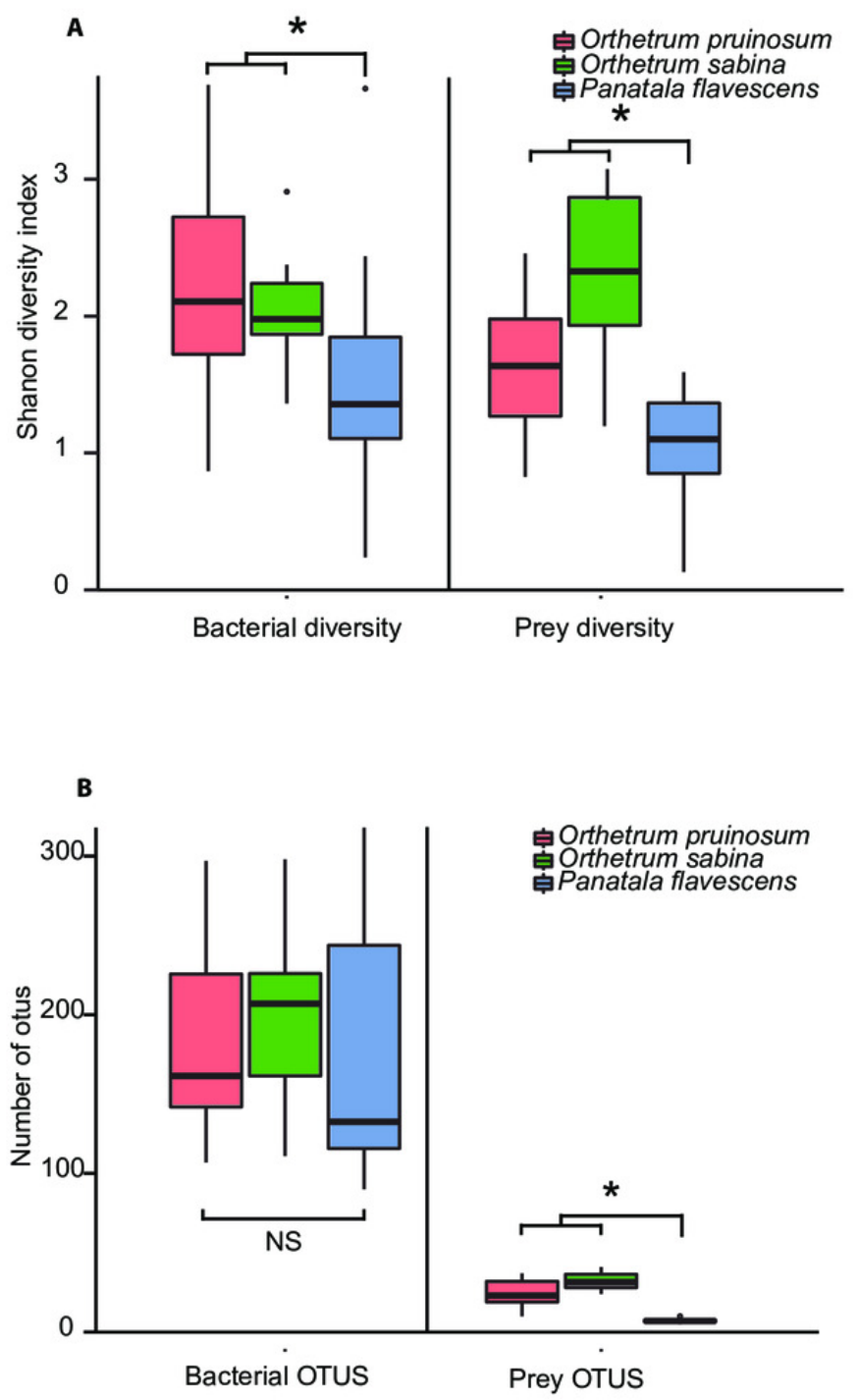

\section{C}

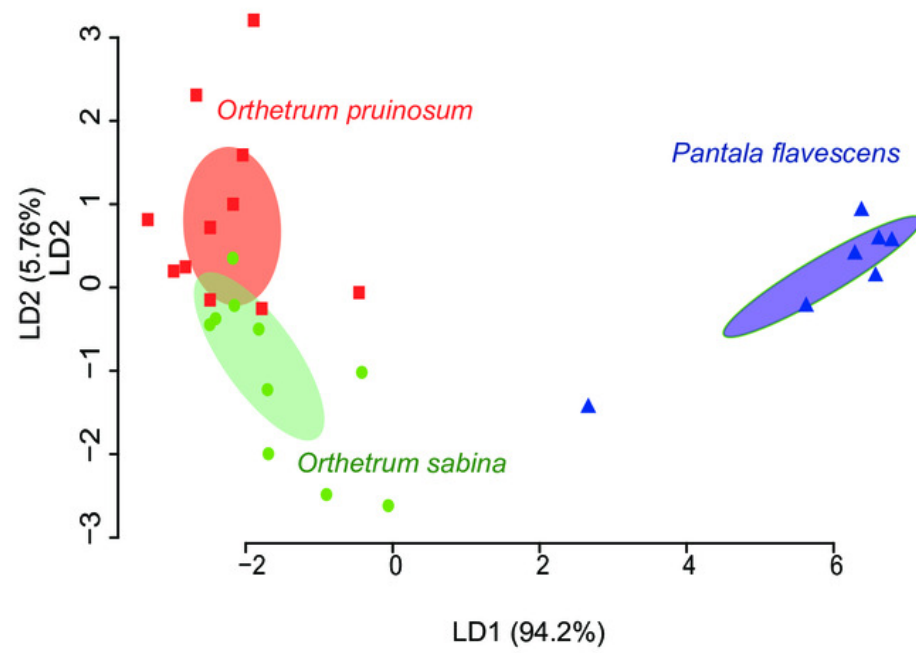


Figure 4

Figure 4

Barplots show the proportion of bacteria whose distribution is consistent with positive selection, neutral assembly, or negative/purifying selection, for dragonflies sampled from a given location.

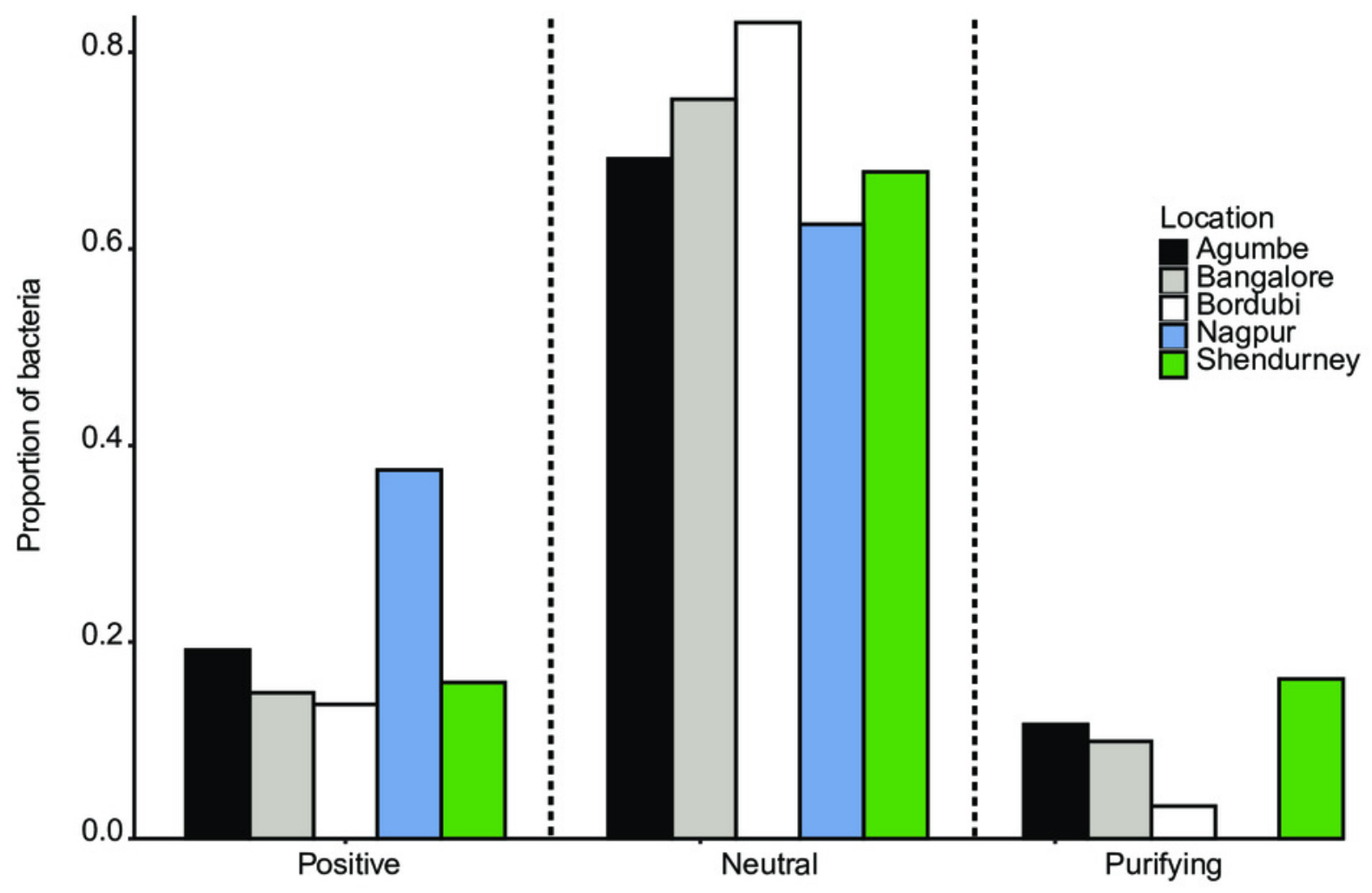




\section{Figure 5}

Figure 5

Examples of Fluorescent in situ hybridisation (FISH) images of dragonfly gut sections using bacteria-specific probes. Host cell nuclei are stained purple with DAPI, eubacteria are green, and Wolbachia is pink. Arrows highlight bacteria in each section. (A) Representative brightfield image of $P$. flavescens midgut section showing columnar cellular folds covering the gut lumen, and food particles in the lumen. (B-D) Foregut sections of $P$. flavescens, $O$. sabina and $O$. pruinosum. Note the lack of eubacterial or Wolbachia signal. (E-G) Midgut and $(\mathrm{H}-\mathrm{J})$ hindgut sections of each species, stained with a eubacterial probe. Note the strong eubacterial signal near the columnar folds of $P$. flavescens. (K-M) Midgut and (N-P) hindgut sections of each species, stained with a Wolbachia-specific probe. Note the lack of signal in $P$. flavescens, a weak signal in $O$. pruinosum, and a large globular structure with Wolbachia in o. sabina. 


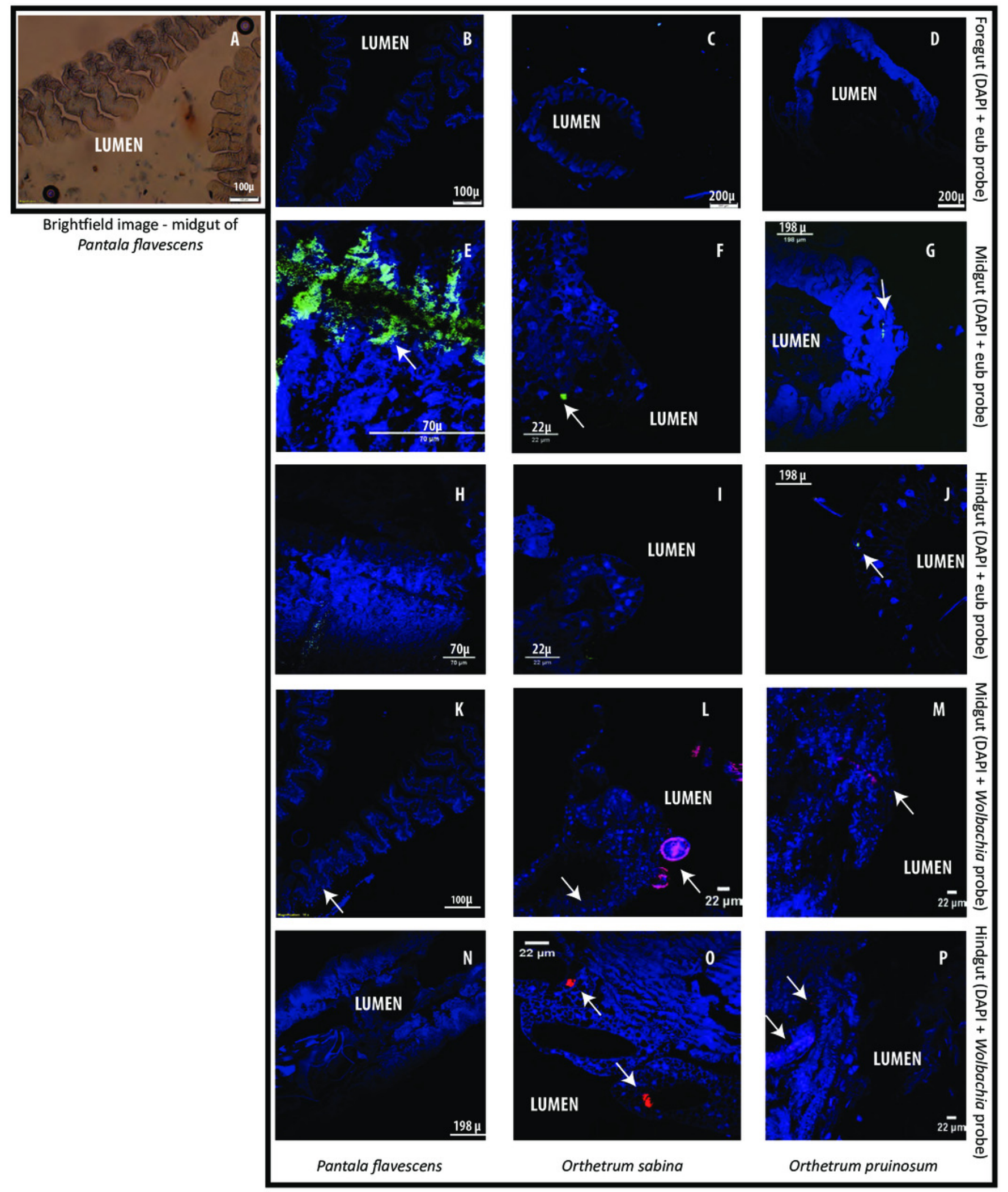


Table $\mathbf{1}$ (on next page)

Tables 1 
1 Table 1. A) Results of a permutational analysis of variation (PERMANOVA) showing the effect

2 of host species and location on gut bacterial composition (open referenced dominant bacterial

3 community) across the three best-sampled dragonfly hosts (Orthetrum pruinosum, Orthetrum

4 sabina, and Pantala flavescens). B) Results of PERMANOVA showing the individual

5 comparisons of 5 host species (Orthetrum pruinosum, Pantala flavescens, Trithemis aurora,

6 Urothemis signata, and Zygonyx iris) based on their gut bacterial composition at a single

7 geographic location - Shendurney.

\begin{tabular}{|c|c|c|c|c|c|c|}
\hline A) & Df & $\mathrm{SSq}$. & Mean SSq. & F stat & $\mathrm{R}^{2}$ & $P$ \\
\hline Species & 2 & 1.31 & 0.66 & 2.36 & 0.09 & 0.005 \\
\hline Location & 4 & 3.77 & 0.94 & 3.40 & 0.26 & $9.9 \times 10^{-5}$ \\
\hline Interaction (Species, Location) & 3 & 1.41 & 0.47 & 1.69 & 0.10 & 0.032 \\
\hline Residuals & 29 & 8.03 & 0.28 & & 0.55 & \\
\hline Total & 38 & 14.52 & & & 1 & \\
\hline B) & Estimate & Std. error & t value & $P$ & & \\
\hline Intercept & 0.11 & 0.09 & 1.15 & 0.28 & & \\
\hline Species (P. flavescens) & -0.26 & 0.12 & -2.09 & 0.07 & & \\
\hline Species (T. aurora) & -0.47 & 0.12 & -3.84 & 0.005 & & \\
\hline Species (U. signata) & 0.33 & 0.13 & 2.47 & 0.04 & & \\
\hline Species (Z. iris) & 0.03 & 0.12 & 0.25 & 0.81 & & \\
\hline
\end{tabular}


Table 2 (on next page)

Table 2 
1 Table 2. (A) Results of a permutational analysis of variation (PERMANOVA) showing the 2 effect of host species on the diet of Orthetrum pruinosum, Orthetrum sabina, and Pantala 3 flavescens. (B) The output of an ordination model (CAPdiscrim) testing the impact of host 4 species on dragonfly diet diversity.

5 A)

\begin{tabular}{lcccccc}
\hline & Df & SSq. & Mean SSq. & F stat & $\mathrm{R}^{2}$ & $\mathrm{P}$ \\
\hline Species & 2 & 3.24 & 1.62 & 8.12 & 0.39 & $9.9 \times 10^{-05}$ \\
Residuals & 25 & 4.99 & 0.20 & & 0.61 & \\
Total & 27 & 8.23 & & & 1 & \\
\hline
\end{tabular}

6

7 B)

\begin{tabular}{lllllll}
\hline Classification success & $75 \%$ & $\mathrm{P}=0.004$ & & & & \\
Proportion of trace & LD1 & LD2 & & & & \\
& 0.94 & 0.057 & & & & \\
\hline Manova & DF & Pillai approx & F & Numerator DF & Denominator DF & P \\
\hline Host Species & 2 & 1.367 & 12.43 & 8 & 46 & $2.78 X$ \\
Residuals & 25 & & & & & $10^{-09}$ \\
\hline
\end{tabular}

8

9

10 